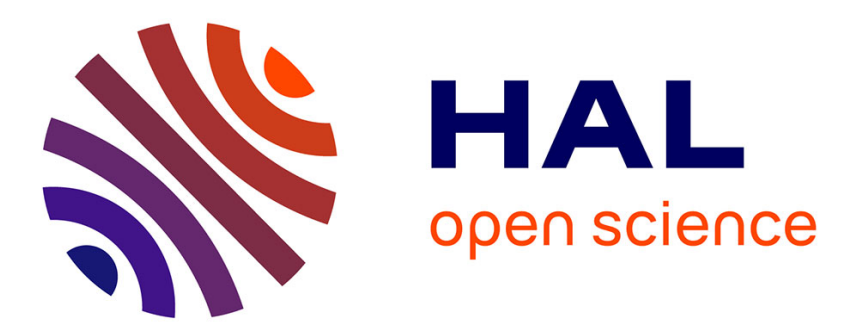

\title{
A coarse-grained transport model for neutral particles in turbulent plasmas
}

\author{
A. Mekkaoui, Yannick Marandet, D. Reiter, P. Boerner, P. Genesio, J. \\ Rosato, H. Capes, M. Koubiti, L. Godbert-Mouret, R. Stamm
}

\section{To cite this version:}

A. Mekkaoui, Yannick Marandet, D. Reiter, P. Boerner, P. Genesio, et al.. A coarse-grained transport model for neutral particles in turbulent plasmas. Physics of Plasmas, 2012, 19 (6), pp.060701. 10.1063/1.4725504. hal-01023293

\section{HAL Id: hal-01023293 https://hal.science/hal-01023293}

Submitted on 11 Jul 2014

HAL is a multi-disciplinary open access archive for the deposit and dissemination of scientific research documents, whether they are published or not. The documents may come from teaching and research institutions in France or abroad, or from public or private research centers.
L'archive ouverte pluridisciplinaire HAL, est destinée au dépôt et à la diffusion de documents scientifiques de niveau recherche, publiés ou non, émanant des établissements d'enseignement et de recherche français ou étrangers, des laboratoires publics ou privés. 


\title{
A coarse-grained transport model for neutral particles in turbulent plasmas
}

\author{
A. Mekkaoui, ${ }^{1}$ Y. Marandet, ${ }^{2, a)}$ D. Reiter, ${ }^{1}$ P. Boerner, ${ }^{1}$ P. Genesio, ${ }^{2}$ J. Rosato, ${ }^{2}$ H. Capes, ${ }^{2}$ \\ M. Koubiti, ${ }^{2}$ L. Godbert-Mouret, ${ }^{2}$ and R. Stamm ${ }^{2}$ \\ ${ }^{1}$ IEK-4 Plasmaphysik, Forschungszentrum Jülich GmbH, Euratom Association, D-52425 Jülich, Germany \\ ${ }^{2}$ Aix-Marseille Université, CNRS, PIIM, UMR 7345, F-13397 Marseille Cedex 20, France
}

(Received 12 September 2012; accepted 28 November 2012; published online 18 December 2012)

\begin{abstract}
The transport of neutral particles in turbulent plasmas is addressed from the prospect of developing coarse-grained transport models which can be implemented in code suites like B2-EIRENE, currently used for designing the ITER divertor. The statistical properties of turbulent fluctuations are described by a multivariate Gamma distribution able to retain space and time correlations through a proper choice of covariance function. We show that in the scattering free case, relevant for molecules and impurity atoms, the average neutral particle density obeys a Boltzmann equation with an ionization rate renormalized by fluctuations. This result lends itself to a straightforward implementation in the EIRENE Monte Carlo solver for neutral particles. Special emphasis is put on the inclusion of time correlations, and in particular on the ballistic motion of coherent turbulent structures. The role of these time dependent effects is discussed for $\mathrm{D}_{2}$ molecules and beryllium atoms. The sensitivity of our results to the assumptions on the statistical properties of fluctuations is investigated. (C) 2012 American Institute of Physics. [http://dx.doi.org/10.1063/1.4771688]
\end{abstract}

\section{INTRODUCTION}

The plasma profiles in the scrape-off-layer (SOL) and divertor are of essential importance to the ITER (and all fusion power plants beyond ITER) design process, since they govern particle and heat fluxes on the plasma facing components, plasma purity, and helium ash removal capabilities. ${ }^{1}$ These profiles are currently predicted with the 2D B2EIRENE (version SOLPS4.3) transport code. ${ }^{2}$ This code, as well as similar codes developed by other groups (e.g., UEDGE, $^{3,4}$ SOLEDGE2D-EIRENE ${ }^{5}$ ), aims at including all physical processes at play in the SOL, i.e., various atomic physics processes (molecular dissociation channels, impurity transport), and plasma wall interactions (sputtering, wall response to the power loading, wall composition changes, and so on). However, achieving this goal requires some coarsegraining in order to obtain a computationally tractable model. In particular, micro-turbulent fluctuations are not resolved. For example, the continuity equation in a transport code reads

$$
\partial_{t}\left\langle n_{e}\right\rangle+\nabla \cdot\left(\left\langle n_{e}\right\rangle\langle\mathbf{v}\rangle+\left\langle\tilde{n}_{e} \tilde{\mathbf{v}}\right\rangle\right)=\left\langle S_{n}\right\rangle,
$$

where $\mathbf{v}$ is the fluid velocity, $\tilde{n_{e}}$ and $\tilde{\mathbf{v}}$ are the fluctuating parts of the density and the velocity fields, $S_{n}$ the particle source, and the brackets $\langle\cdot\rangle$ stand for a time average. The latter introduces a turbulent contribution to the particle flux $\boldsymbol{\Gamma}_{\text {turb }}=\left\langle\tilde{n_{e}} \tilde{\mathbf{v}}\right\rangle$ in the equations for the plasma fluid, which has to be expressed in terms of the coarse grained density field $\left\langle n_{e}\right\rangle$ in order to close the equation. The turbulent flux is generally modeled by a diffusive flux, namely $\Gamma_{\text {turb }}=-D_{a} \boldsymbol{\nabla}\left\langle n_{e}\right\rangle$, where $D_{a}$ is the anomalous diffusion coefficient. However, SOL turbulence is characterized by coherent structures propagating ballistically through the SOL, termed "blobs," "filaments," or "avalanches."7 The

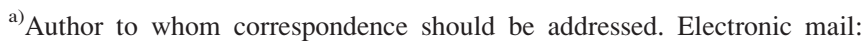
yannick.marandet@univ-amu.fr.
}

transport resulting from the propagation of these structures is not necessarily well described by a diffusion process so that it has been proposed to add a convective flux $\mathrm{v}_{a}\left\langle n_{e}\right\rangle$ to the turbulent flux, ${ }^{8}$ introducing a second "anomalous" parameter, the advection velocity $\mathrm{v}_{a}$. But in order to obtain a truly consistent set of coarse-grained transport equations, $S_{n}$ (and any other terms in the momentum or energy equation) should be properly averaged over fluctuations. This is especially true in SOL plasmas, which exhibit intermittent turbulence with large relative fluctuation levels, up to order unity in the far SOL for the plasma density $n_{e} .{ }^{9}$ Indeed, if $g$ is a non-linear function of $X$, then $\langle g(X)\rangle \neq g(\langle X\rangle) .{ }^{10-16}$ This explains the differences (on radiated power, sputtering rates, etc.), between a diffusive/convective description of the turbulent flux and an improved model including the dynamics of coherent structures, at a higher computational cost. ${ }^{17,18}$ In this work, we focus on the average of the source terms in the equations for plasma density, momentum and energy conservation, and present a coarse-grained kinetic equation for neutral particles, exact within a set of physically relevant assumptions. These assumptions are presented in Sec. II. Fluctuations are described by a multivariate Gamma distribution (MGD), whose two-point covariance function can be set from experiments and/or numerical simulations. New results on the higher order statistical properties of the MGD are given. In Sec. III, we derive the coarse-grained kinetic equation satisfied by the neutral particle phase space distribution, in two different limiting cases for the temporal response of the recycling flux to the impinging plasma flux. The results first presented in a letter ${ }^{19}$ are generalized in several ways. The ballistic propagation of coherent structures is now explicitly included in the model, through a proper choice of covariance function. Applications in a 2D slab geometry with the EIRENE code ${ }^{2}$ show that the motion of structures can play a role for molecules, i.e., it can weaken the effects of fluctuations on neutral particle transport, but 
that the effect on neutral beryllium transport is essentially negligible. For an exponential covariance function and homogenous statistics, a new exact expression for the coarsegrained ionization rates is obtained in terms of elementary functions. This result provides very useful checks on all numerical calculations. Finally, in Sec. IV we discuss the sensitivity of our results to the choice of the MGD to describe fluctuations. We first investigate the differences on the transport of neutral particles resulting from the choice of multivariate Log-normal statistics instead of MGD statistics. Then, using the results obtained in Sec. II, we modify the MGD in such a way that the probability density function (PDF) at one point in space and the covariance function are unchanged, since these quantities can directly be related to experiments/simulations. The resulting changes on the average neutral density are then investigated.

\section{MODEL ASSUMPTIONS}

\section{A. Boltzmann equation in the scattering-free limit}

Our previous works on the transport of neutral particles in the presence of density fluctuations have clearly shown that in order for significant effects to arise, the neutral particle mean free path must not be much larger than the turbulence correlation length. ${ }^{19,22,23}$ As a result, in typical SOL conditions the effects of fluctuations on fuel atoms (hydrogen isotopes) can be safely ignored. This is true even for fuel atoms thermally released from the wall, because of resonant charge exchange. ${ }^{20}$ Here, we focus on molecules and impurity atoms, for which charge exchange or elastic collisions can usually be neglected in SOL conditions (that is, the transport problem is, essentially, scattering-free). From the neutrals point of view, plasma fluctuations translate into fluctuations of the ionization (or molecular dissociation) rate, $\nu(\mathbf{r}, t)=n_{e}(\mathbf{r}, t) \overline{\sigma_{i o} \mathrm{~V}_{e}}$, where $\overline{\sigma_{i o} \mathrm{~V}_{e}}$ is the rate coefficient. In the following, we consider only density fluctuations and assume that the ionization rate coefficient does not depend on density (corona limit) so that density and ionization rate have the same statistical properties up to a scaling factor. This is a rather mild approximation. It corresponds to a low density limit, which can be regarded as valid in the far scrape-off layer (less so in the divertor). In addition, the dependence of the ionization rate coefficient on the neutral particle velocity $\mathbf{v}_{0}=\mathrm{v}_{0} \boldsymbol{\Omega}$ is usually negligible for electron impact processes, since $\left|\mathbf{v}_{0}-\mathbf{v}_{e}\right| \simeq\left|\mathbf{v}_{e}\right|$ owing to the much larger electron velocity $\mathbf{v}_{e}$. The transport equation for the neutrals is of the Boltzmann type, and reads

$$
\left[\partial_{t}+\mathrm{v}_{0} \boldsymbol{\Omega} \cdot \boldsymbol{\nabla}+\nu(t, \mathbf{r})\right] f\left(t, \mathbf{r}, \mathrm{v}_{0}, \boldsymbol{\Omega}\right)=0 .
$$

The analytical solution of this equation, assuming the initial condition $f\left(t=0, \mathbf{r}, \mathrm{v}_{0}, \boldsymbol{\Omega}\right)$ is zero, is (e.g., Ref. 21)

$$
\begin{aligned}
f\left(t, \mathbf{r}, \mathrm{v}_{0}, \boldsymbol{\Omega}\right)= & \Gamma_{0}\left(t-\frac{\left|\mathbf{r}-\mathbf{r}_{w}\right|}{\mathrm{v}_{0}}, \boldsymbol{\Omega}, \mathrm{v}_{0}\right) \\
& \times \exp \left(-\frac{1}{\mathrm{v}_{0}} \int_{0}^{\left|\mathbf{r}-\mathbf{r}_{w}\right|} \nu\left(\mathbf{r}-s \boldsymbol{\Omega}, t-s / \mathrm{v}_{0}\right) d s\right) \\
& \times \frac{\Theta\left(\mathrm{v}_{0} t-\left|\mathbf{r}-\mathbf{r}_{w}\right|\right)}{\mathrm{v}_{0}},
\end{aligned}
$$

where $\Gamma_{0}$ is the neutral particle flux density at the wall, located at $\mathbf{r}_{w}$, and $\Theta$ the Heaviside step function. The latter was discarded in Ref. 19 because there we considered only times such that $t \gg\left|\mathbf{r}-\mathbf{r}_{w}\right| / \mathrm{v}_{0}$, not the initial relaxation of the system. The optical depth $\tau$ is defined as the modulus of the argument of the exponential in Eq. (3), namely

$$
\tau\left(\left|\mathbf{r}-\mathbf{r}_{w}\right|, \boldsymbol{\Omega}, t\right)=\frac{1}{\mathrm{v}_{0}} \int_{0}^{\left|\mathbf{r}-\mathbf{r}_{w}\right|} \nu\left(\mathbf{r}-s \boldsymbol{\Omega}, t-s / \mathrm{v}_{0}\right) d s .
$$

The neutral particle density is obtained by integrating over the source velocity distribution $F\left(t, \mathrm{v}_{0}, \mathbf{\Omega}\right)$, namely

$$
N(\mathbf{r}, t)=\int_{4 \pi} d^{2} \boldsymbol{\Omega} \int_{0}^{+\infty} \mathrm{v}_{0}^{2} d \mathrm{v}_{0} F\left(t, \mathrm{v}_{0}, \boldsymbol{\Omega}\right) f\left(t, \mathbf{r}, \mathrm{v}_{0}, \boldsymbol{\Omega}\right)=\{f\},
$$

where the curly brackets $\{\cdot\}$ stand for the velocity average. The adiabatic approximation on which we relied in Refs. 22 and 23 consists in neglecting the time delay $s / \mathrm{v}_{0}$ in Eq. (3). Within this approximation, Eq. (3) obeys Eq. (2) with the time derivative neglected, that is, the neutral velocity distribution follows instantaneously the evolution of the plasma parameters. In other words, the plasma density does not have time to evolve significantly during the typical lifetime of a neutral particle. The adiabatic regime is valid provided $n_{e} / \partial_{t} n_{e} \gg \max \left(1 / \overline{n_{e}} \overline{\sigma_{i o} \mathrm{v}_{e}},\left|\mathbf{r}-\mathbf{r}_{w}\right| / \mathrm{v}_{0}\right)$, where $\overline{n_{e}}$ is the line averaged density along the neutral flight. Taking $n_{e} / \partial_{t} n_{e}$ as the correlation time of turbulence $\tau_{\text {turb }}$, that is $\tau_{\text {turb }} \simeq 10 \mu \mathrm{s}$ (Ref. 6), $\overline{n_{e}}=5 \times 10^{18} \mathrm{~m}^{-3}$ and $\overline{\sigma_{i o} \mathrm{~V}_{e}}=10^{-13} \mathrm{~m}^{3} \cdot \mathrm{s}^{-1}$, yields $\tau_{\text {turb }} / \tau_{i o} \simeq 5 \quad\left(\tau_{i o}=1 / \overline{h_{e}} \overline{\sigma_{i o} \mathrm{v}_{e}}\right)$. The requirements are more stringent for the deep penetration problem, where $\left|\mathbf{r}-\mathbf{r}_{w}\right|$ is several neutral mean free paths. As a result, the adiabatic approximation is only marginally valid according to the criteria stated above.

The neutral density is closely related to the neutral particle flux through a given surface $\mathscr{S}$, which is given by

$$
\Gamma=\iint_{\mathscr{S}}\left\{f\left(t, \mathbf{r}, \mathbf{v}_{0}, \boldsymbol{\Omega}\right) \mathbf{v}_{0}\right\} \cdot d \mathbf{S} .
$$

This flux is an important quantity, as far as, e.g., impurity penetration, helium pumping or sputtering by impurities are concerned. The source/sink terms corresponding to exchanges of particles, momentum (ignoring electron momentum because of the smallness of the ratio of the electron to the ion masses), and energy between the neutrals and the plasma are given by

$$
\begin{gathered}
S_{n}(\mathbf{r}, t)=\nu(\mathbf{r}, t) N(\mathbf{r}, t), \\
\mathbf{S}_{m}(\mathbf{r}, t)=\nu(\mathbf{r}, t)\left\{m \mathbf{v}_{0} f\left(t, \mathbf{r}, \mathrm{v}_{0}, \mathbf{\Omega}\right)\right\}, \\
S_{E_{s}}(\mathbf{r}, t)=\nu(\mathbf{r}, t)\left\{\left[\frac{1}{2} m_{s} \mathbf{v}_{0}^{2}-E_{s}^{l}\right] f\left(t, \mathbf{r}, \mathbf{v}_{0}, \boldsymbol{\Omega}\right)\right\},
\end{gathered}
$$

where $m$ is the atom mass, $s=i, e$ stands for the species (electrons or ions), and $E_{s}^{l}$ is the energy cost per ionization (or dissociative ionization for molecules). $E_{e}^{l}$ includes radiative losses, and $E_{i}^{l}=0$. Note that $E_{e}^{l}$ is independent on the density, in accordance with our assumptions for the 
ionization rate coefficient (corona limit). These expressions for the sources are valid when all absorption channels for the neutral particles release one electron. A more general expression will be given in Sec. III C. When scattering is negligible, the Boltzmann equation can be solved independently for each direction $\boldsymbol{\Omega}$ of the initial neutral particle velocity, and for a given value of $v_{0}$, see Eq. (3). As a consequence, in the following we will often work in a 1D geometry along the neutral trajectory. However, it should be stressed that the results we will present can equally well be applied in $2 \mathrm{D}$ or 3D geometries using Eqs. (5)-(9), as illustrated in Sec. III.

\section{B. Multivariate Gamma distribution}

As stated in Sec. I, we are mainly interested in time averages of the neutral particle flux and of the source terms defined by Eqs. (7)-(9), since these are the relevant quantities on the transport time scale. Calculating these averages requires the knowledge of the probability density functional of the density field $n_{e}(\mathbf{r}, t)$ ( since $\left.\nu(\mathbf{r}, t)=n_{e}(\mathbf{r}, t) \overline{\sigma_{i o} \mathrm{v}_{e}}\right)$ along the neutral trajectory, see Eq. (3). Therefore, we discretize space along the trajectory with a grid step $\epsilon$ so that it is divided in $k$ segments with $s=k \epsilon$. The statistics of the discretized field is given by a multivariate PDF $W\left(n_{e 1}, \ldots, n_{e k}\right)$ $=W\left(\mathbf{n}_{e}\right)$. In the non-adiabatic case, the plasma density field $n_{e j}$ at $s=j \epsilon$ has to be taken at time $t-s / \mathrm{v}_{0}$, to account for the time neutrals need to travel from the source located at $\mathbf{r}_{w}$ to the current point. In our previous works, we took $W$ as a multivariate Gamma distribution (MGD). This choice is justified by measurements of density fluctuations for instance in the TCV tokamak, ${ }^{24}$ whose one-point statistics is well described by a Gamma distribution, i.e.

$$
W_{1}\left(n_{e i}\right)=\frac{n_{e i}^{\beta-1} e^{-n_{e i} / \alpha_{i}}}{\alpha_{i}^{\beta} \Gamma(\beta)} \text { for } n_{e i}>0, \quad \text { and } 0 \text { otherwise }
$$

with $\beta$ the shape factor and $\alpha_{i}$ the scale factor (the relationships between these parameters and others used subsequently in this work are summarized in Appendix A). The Gamma distribution often occurs in statistical studies of turbulence, as pointed out in Ref. 24, and an argument based on the quadratic nonlinearity of the equations describing density fluctuations could explain why the statistics should be Gamma. ${ }^{25}$ Furthermore, it has been shown recently using a stochastic model built from observations made on numerical simulations and experimental data that Gamma statistics can be seen as resulting from the properties of a superposition of independent density bursts (with a prescribed waveform, and exponentially distributed amplitudes). ${ }^{26}$ In the case where $\beta=M / 2, M$ being an integer (the number of degrees of freedom), the distribution defined by Eq. (10) is the marginal of the so-called MGD, ${ }^{27}$ that is

$$
W_{1}\left(n_{e i}\right)=\int \ldots \int\left(\prod_{j \neq i} d n_{e j}\right) W\left(n_{e 1}, \ldots, n_{e k}\right) .
$$

In the following, we will mostly deal with averages of exponentials of the plasma density $n_{e}$. The latter averages are closely related to the moment generating function $Z(\mathbf{u})$ of the MGD, i.e., the multi-dimension Fourier transform of $W$, which is given by

$$
Z(\mathbf{u})=\left\langle\exp -\mathbf{u} \cdot \mathbf{n}_{e}\right\rangle=|\mathbf{I}+2 \mathbf{G} \mathbf{U}|^{-M / 2},
$$

where $\mathbf{u}=\left(u_{1}, \ldots, u_{k}\right)^{\dagger}, \mathbf{I}$ is the $k \times k$ identity matrix, and the matrix $\mathbf{U}$ has entries $U_{i j}=u_{i} \delta_{i j}$. The matrix $\mathbf{G}$ is related to the correlation matrix $C_{i j}$ of the density field, defined by $C_{i j}=\left\langle n_{e i} n_{e j}\right\rangle-\left\langle n_{e i}\right\rangle\left\langle n_{e j}\right\rangle$, through $G_{i j}^{2}=C_{i j} /(2 M)$. As a result, the MGD is fully defined by the spatio-temporal covariance $C\left(\mathbf{r}, \mathbf{r}^{\prime}, t, t^{\prime}\right)$ of the density field, which can be determined either experimentally (e.g., Ref. 28) or numerically (e.g., Ref. 7). Equation (12) with $u_{i}=\epsilon / \mathrm{v}_{0}$ gives the average of Eq. (3), provided the entries $C_{i j}$ are defined by

$$
C_{i j}=C\left(\mathbf{r}_{i}, \mathbf{r}_{i}+\Delta s \boldsymbol{\Omega}, t_{i}, t_{i}+\Delta s / \mathrm{v}_{0}\right)=C_{L}\left(\Delta s, \mathbf{v}_{0}, \mathbf{r}_{i}, t_{i}\right),
$$

where $\Delta s=\left(\mathbf{r}_{j}-\mathbf{r}_{i}\right) \cdot \boldsymbol{\Omega}$. The function $C_{L}$ will be called the Lagrangian covariance in the following, because it represents correlations along the neutral particle trajectory. In the homogenous and stationary case

$$
C_{i j}=C\left(\Delta s \boldsymbol{\Omega}, \Delta s / \mathrm{v}_{0}\right)=C_{L}\left(\Delta s, \mathbf{v}_{0}\right) .
$$

Note that the adiabatic approximation can be stated as $C\left(\mathbf{r}, \mathbf{r}^{\prime}, t, t^{\prime}\right) \simeq C\left(\mathbf{r}, \mathbf{r}^{\prime}, t, t\right)$, in which case $C_{L} \simeq C$. The parameter $M$ determines the relative fluctuation level $R$ $=\sigma_{n_{e}} /\left\langle n_{e}\right\rangle \quad\left(\sigma_{n_{e}}^{2}\right.$ is the local variance of the fluctuations), namely $R=\sqrt{2 / M}=1 / \sqrt{\beta}$. Strictly speaking, the MGD is only defined for integer values of $\mathbf{M}$, and $W_{1}(0)=0$ requires $M \geq 3(R<82 \%)$. However, if the characteristic function $Z(\mathbf{u})$ is infinitely divisible (that is, $Z$ can be written as $Z=\left(Z_{n}\right)^{n}$, where $Z_{n}$ is a characteristic function for all values of the integer $n$ (Ref. 29)), the MGD can be defined for positive real values of $\beta .^{30}$ Infinite divisibility holds provided the correlation matrix $\zeta$, defined by $\zeta_{i j}=C_{i j} /\left(\sigma_{n_{e i}} \sigma_{n_{e j}}\right)$ is such that $\zeta_{i j}=\zeta_{i k} \zeta_{k j}$, with $i \leq k \leq j$, ${ }^{31}$ a relation which is satisfied by the exponential function. As a result, the MGD can be extended to any real value of $\beta=M / 2$ if the covariance function is exponential.

\section{Covariances}

One of the appealing features of a stochastic description of fluctuations is the possibility to investigate various model cases, by scanning the input parameters for the model, that is $\beta=M / 2$ and the covariance $C$. The latter must however be positive definite, in order to ensure the realizability of the stochastic model. In fact, if $\mathrm{C}$ is not positive definite, it is possible to construct a linear combinations of the $n_{e i}$ that has a negative variance, ${ }^{33}$ hence a "PDF" with negative values (the corresponding multivariate distribution is then said to be non-realizable ${ }^{32}$ ). An equivalent way of defining positivedefiniteness is to require that the Fourier transform of the covariance function (that is, the power spectrum according to the Wiener-Khinchin theorem ${ }^{33}$ ) be positive. This is in practice a major constraint on the choice of $\mathrm{C}$. In the following, we will consider the following anisotropic covariance function: 


$$
\begin{aligned}
C\left(\mathbf{r}-\mathbf{r}^{\prime}, t-t^{\prime}\right) & \\
= & \sigma_{n_{e}}^{2} \exp \left(-\left[\sum_{\alpha=x, y}\left(\frac{x_{\alpha}-x_{\alpha}^{\prime}-\mathrm{v}_{s \alpha}\left(t-t^{\prime}\right)}{\lambda_{\alpha}}\right)^{2}\right]^{1 / 2}\right) \\
& \times \exp \left(-\frac{\left|t-t^{\prime}\right|}{\tau_{d}}\right),
\end{aligned}
$$

where the propagation of coherent structures is accounted for by the velocity $\mathbf{v}_{s}$ (see Ref. 28 for a similar discussion), and $\tau_{d}$ is the lifetime of the structures. When $\mathbf{v}_{s}=0$, the covariance is separable in the sense that the space and time dependences can be factorized. The turbulence correlation length $\lambda$ (in direction $\boldsymbol{\Omega}$ ) is defined by

$$
\lambda(\boldsymbol{\Omega})=\frac{1}{\sigma_{n_{e}}^{2}} \int_{0}^{+\infty} C(s \boldsymbol{\Omega}, 0) d s=\left(\frac{\mu^{2}}{\lambda_{x}}+\frac{\sqrt{1-\mu^{2}}}{\lambda_{y}}\right)^{-1 / 2},
$$

with $\mu=\cos \theta=\boldsymbol{\Omega} \cdot \mathbf{u}_{x}$. In the same way, the correlation time of the fluctuations is

$$
\tau_{\text {turb }}=\frac{1}{\sigma_{n_{e}}^{2}} \int_{0}^{+\infty} C(\mathbf{0}, t) d t=\left(\frac{\left|\mathrm{v}_{s x}\right|}{\lambda_{x}}+\frac{\left|\mathrm{v}_{s y}\right|}{\lambda_{y}}+\frac{1}{\tau_{d}}\right)^{-1} .
$$

When $\mathbf{v}_{s}=\mathbf{0}, \tau_{\text {turb }}=\tau_{d}$, but if for instance $\mathbf{v}_{s y}=0$ and $\lambda_{x} /\left|\mathrm{v}_{s x}\right| \ll \tau_{d}$, decorrelation essentially occurs because of the movement of the structures and $\tau_{\text {turb }} \simeq \lambda_{x} /\left|\mathrm{v}_{s x}\right|$. As an illustration, we consider on Fig. 1 the density field $n_{e}(x, t)$ along the line such that $\mathbf{\Omega}=\mathbf{u}_{x}$, with $\lambda_{x}=10, \tau_{d}=20$ and (a) $\mathrm{v}_{s x}=0$, (b) $\mathrm{v}_{s x}=1$, in arbitrary units. These plots show the ability of our model to capture the propagation of coherent structures.

For the covariance of Eq. (15), the characteristic function of the MGD, given by Eq. (12), can be expressed in terms of elementary functions. Taking the continuous limit in a formula derived by Grenander and Szegö ${ }^{34}$ for the determinant of the matrix $\mathbf{A}=\mathbf{I}+2 \mathbf{G U}$ leads to (see Appendix B)

$$
Z_{e}(u)=\frac{\exp \left(\frac{\beta s}{2 \lambda_{L}}\right)}{\left[\cosh \left(\frac{g s}{2 \lambda_{L}}\right)+\frac{g^{2}+1}{2 g} \sinh \left(\frac{g s}{2 \lambda_{L}}\right)\right]^{\beta}},
$$
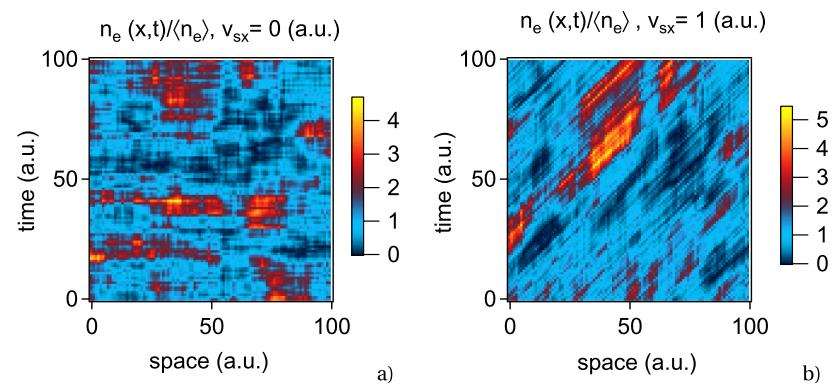

FIG. 1. Space-time plot of a realizations of the plasma density along the direction $\boldsymbol{\Omega}=\mathbf{u}_{x}$, with $\lambda_{x}=20, \tau_{d}=10$ and (a) $\mathrm{v}_{s x}=0$ and (b) $\mathrm{v}_{s x}=1$, in arbitrary units. In the latter case, the turbulent structures move along the $x=\mathrm{v}_{s x} t$ line. with $g(u)=\sqrt{1+4 \lambda_{L}\left\langle n_{e}\right\rangle u / \beta}, u$ a scalar, and $\lambda_{L}$ the Lagrangian correlation length defined by $\lambda_{L}=\int_{0}^{+\infty} C_{L}(s) d s / C(0)$. The latter is given by

$$
\lambda_{L}=\frac{\mathrm{v}_{0} \tau_{d} \lambda}{\lambda+\mathrm{v}_{0} \tau_{d}\left|1-\mathbf{v}_{s} \cdot \mathbf{\Omega} / \mathrm{v}_{0}\right|},
$$

assuming for simplicity $\lambda_{x}=\lambda_{y}=\lambda$. Properly speaking, in the adiabatic case $Z_{e}(u)$ is the characteristic function of the line averaged density $\int_{0}^{s} n_{e}\left(s^{\prime}, t\right) d s^{\prime}$. This simple formula provides a strong check on all the numerical calculations performed in the following. Other possible choices for homogeneous and stationary covariances can be found in, e.g., Refs. 35-37. For non-homogeneous and non-stationary cases, examples can be found in Refs. 35-40.

\section{Higher order statistics}

As stated in Sec. II B, the MGD is fully specified by its covariance and the value of the parameter $\beta=M / 2$. The 1-point PDF and the covariance obtained from experiments or numerical simulations therefore completely determine the statistical properties of fluctuations within our model. However, there is no guarantee that the r-point moments $\left\langle n_{e}\left(\mathbf{r}_{1}\right) \ldots n_{e}\left(\mathbf{r}_{r}\right)\right\rangle, r>2$, are consistent with those resulting from the MGD. The sensitivity of our results to these higher order statistical quantities should therefore be quantified. In this section, we consider the optical depth $\tau$ defined by Eq. (4), which is the most relevant quantity as far as the transport of neutral particles is concerned. In addition, we work with the so-called cumulants instead of moments, because the former have simpler expressions. While moments can be obtained from successive differentiations of the moment generating function (or characteristic function) $Z(u)=\left\langle e^{-u \tau}\right\rangle$, cumulants are defined in terms of $\psi(u)=\ln Z(u)$, the latter being called the cumulant generating function. The rth order cumulant of the optical depth is defined by

$$
\left\langle\left\langle\tau^{r}\right\rangle\right\rangle=\left.(-1)^{r} \frac{d^{r} \psi}{d u^{r}}\right|_{u=0} .
$$

We have $\langle\langle\tau\rangle\rangle=\langle\tau\rangle$, while the second cumulant is nothing but the variance, namely $\left\langle\left\langle\tau^{2}\right\rangle\right\rangle=\left\langle\tau^{2}\right\rangle-\langle\tau\rangle^{2}$. The third and fourth order cumulants are, respectively, related to the skewness $S=\left\langle\left\langle\tau^{3}\right\rangle\right\rangle /\left\langle\left\langle\tau^{2}\right\rangle\right\rangle^{3 / 2}$ and the normalized kurtosis $K=\left\langle\left\langle\tau^{4}\right\rangle\right\rangle /\left\langle\left\langle\tau^{2}\right\rangle\right\rangle^{2}$. For the MGD, the cumulants can be calculated exactly (Appendix C), namely

$$
\begin{aligned}
\left\langle\left\langle\tau(s, \boldsymbol{\Omega})^{r}\right\rangle\right\rangle= & \frac{2^{r}(r-1) ! \beta}{\left(4 \beta \mathrm{v}_{0}^{2}\right)^{r / 2}} \int_{0}^{s} d s_{1} \ldots \int_{0}^{s} d s_{r} \\
& \times\left[C_{L}(1,2) C_{L}(2,3) \ldots C_{L}(r-1, r) C_{L}(r, 1)\right]^{1 / 2},
\end{aligned}
$$

where $C_{L}(i, j)=C\left(\mathbf{r}_{w}+s_{i} \boldsymbol{\Omega}, \mathbf{r}_{w}+s_{j} \boldsymbol{\Omega}, t-s_{i} / \mathrm{v}_{0}, t-s_{j} / \mathrm{v}_{0}\right)$ is the Lagrangian covariance function. The fluctuations are assumed to be stationary so that the cumulants are independent of time. We provide here the expressions of the cumulants for the covariance defined by Eq. (15) up to order $r=4$, obtained from a direct calculation of the $r$ th dimensional integral in Eq. (21). The result is 


$$
\begin{aligned}
&\left\langle\left\langle\tau(s, \boldsymbol{\Omega})^{2}\right\rangle\right\rangle=-\frac{2}{\beta}\left(\frac{\lambda_{L}}{\ell}\right)^{2}\left[1-\frac{s}{\lambda_{L}}-e^{-s / \lambda_{L}}\right], \\
&\left\langle\left\langle\tau(s, \boldsymbol{\Omega})^{3}\right\rangle\right\rangle=-\frac{12}{\beta^{2}}\left(\frac{\lambda_{L}}{\ell}\right)^{3}\left[2-\frac{s}{\lambda_{L}}-\left(2+\frac{s}{\lambda_{L}}\right) e^{-s / \lambda_{L}}\right], \\
&\left\langle\left\langle\tau(s, \boldsymbol{\Omega})^{4}\right\rangle\right\rangle= \frac{24}{\beta^{3}}\left(\frac{\lambda_{L}}{\ell}\right)^{4} e^{-s / \lambda_{L}}\left[14+10 \frac{s}{\lambda_{L}}+2\left(\frac{s}{\lambda_{L}}\right)^{2}\right. \\
&+\left(5 \frac{s}{\lambda_{L}}-14\right) \cosh \left(-\frac{s}{\lambda_{L}}\right) \\
&\left.+\left(5 \frac{s}{\lambda_{L}}-3\right) \sinh \left(-\frac{s}{\lambda_{L}}\right)\right]
\end{aligned}
$$

where we recall that $\ell=\mathrm{v}_{0} /\left(\left\langle n_{e}\right\rangle \overline{\sigma_{i o} \mathrm{v}_{e}}\right)$, and where $\lambda_{L}$ is the Lagrangian correlation length. These expressions are in full agreement with those obtained from successive derivations of the cumulant generating function $\ln Z_{e}(u)$, where $Z_{e}(u)$ is defined by Eq. (18). In addition, they have the expected behavior in different limiting cases. For $s / \lambda_{L} \ll 1$, the r-dimensional integral reduces to $s^{r}$, that is the volume of the hypercube of side length $s$, while for $s / \lambda_{L} \gg 1$ we obtain $\left\langle\left\langle\tau^{r}\right\rangle\right\rangle \propto \lambda_{L}^{n-1} s$, as expected from well known properties of the cumulants (see, e.g., Ref. 41, p. 405).

Equation (21) shows that the behavior of the cumulants of $\int_{0}^{s} n_{e} d s_{1} \propto \tau(s)$, a quantity easily computable in numerical simulations, as a function of $s$ can be used to check whether the higher order statistical properties of fluctuations are consistent with those resulting from MGD statistics. The sensitivity of the average neutral density to the form of these cumulants for $r>2$ will be investigated in Sec. IV A.

\section{COARSE-GRAINED TRANSPORT EQUATION}

We now look for the transport equation satisfied by $\langle f\rangle$, in the light of the results summarized in Sec. II. Averaging Eq. (2) over density fluctuations leads to

$$
\left[\partial_{t}+\mathrm{v}_{0} \mathbf{\Omega}\right]\langle f\rangle+\left\langle\nu(t, \mathbf{r}) f\left(t, \mathbf{r}, \mathrm{v}_{0}, \boldsymbol{\Omega}\right)\right\rangle=0,
$$

where a part of the crossed term, namely $\langle\tilde{\nu} \tilde{f}\rangle$, plays the same role as the turbulent flux in Eq. (1). This equation is supplemented by a boundary condition at $s=0$, which depends on the recycling model. In Refs. 19, 22, and 23, we considered two limiting cases defined by the ordering between the typical turbulent time scales $\tau_{\text {turb }}$ and the recycling time scales $\tau_{R}$. The slow recycling case is defined by $\tau_{R} \gg \tau_{\text {turb }}$, a situation where the recycling flux is constant (assuming stationary turbulence) and equal to the time averaged plasma flux (assuming saturated walls). This model might provide a reasonable description for molecules desorbed from the wall. The opposite case, where $\tau_{R} \ll \tau_{\text {turb }}$, is termed "fast recycling" and describes a situation where the wall has an instantaneous response so that the recycling flux follows the plasma flux. $\Gamma_{0}$ is then a random variable, and we shall assume that $\Gamma_{0} \propto n_{e}\left(\mathbf{r}_{w}\right)$. Back-scattered atoms and physically sputtered impurities are well described by this model, given the short time scales involved. In the following, we will again consider these two limiting cases.

\section{A. Slow recycling}

The particle source defined by Eq. (7) is calculated by functional differentiation of Eq. (12), i.e.

$$
\begin{aligned}
\left\langle\nu(\mathbf{r}, t) f^{S}\left(t, \mathbf{r}, \mathrm{v}_{0}, \boldsymbol{\Omega}\right)\right\rangle & =-\left.\frac{\left\langle\Gamma_{0}\right\rangle \overline{\sigma_{i o} \mathbf{v}_{e}}}{\mathbf{v}_{0}} \frac{\delta Z[u]}{\delta u\left(\left|\mathbf{r}-\mathbf{r}_{w}\right|\right)}\right|_{u=u_{c}} \\
& =\varpi^{S}\left(\left|\mathbf{r}-\mathbf{r}_{w}\right|\right)\left\langle f^{S}\right\rangle,
\end{aligned}
$$

where $u_{c}=\langle\nu\rangle /\left(\mathrm{v}_{0}\left\langle n_{e}\right\rangle\right)=\overline{\sigma_{i o} \mathrm{v}_{e}} / \mathrm{v}_{0}$, and where the quantity

$$
\varpi^{S}\left(\left|\mathbf{r}-\mathbf{r}_{w}\right|\right)=\lim _{k \rightarrow+\infty} \frac{\beta \mathrm{v}_{0} k}{\left|\mathbf{r}-\mathbf{r}_{w}\right|}\left[1-\left(\mathbf{A}_{k}^{-1}\right)_{k k}\right],
$$

with $\mathbf{A}_{k}=\mathbf{I}+2\left|\mathbf{r}-\mathbf{r}_{w}\right| /\left(\mathrm{v}_{0} k\right) \mathbf{G}$, has the dimension of the inverse of a time. We recall that $k$ is the number of discretization steps on the length $\left|\mathbf{r}-\mathbf{r}_{w}\right|$ of the trajectory (see Sec. II B), and $k \rightarrow \infty$ thus corresponds to the continuous limit. The ${ }^{S}$ superscript stands for "slow recycling," and the entries of the $k \times k$ matrix $\mathbf{G}$ are such that

$$
G_{i j}=\left[\frac{1}{4 \beta} C_{L}\left(|i-j| s / k, \mathbf{v}_{0}, \mathbf{r}_{i}\right)\right]^{1 / 2},
$$

with $s=\left|\mathbf{r}-\mathbf{r}_{w}\right|$, and $C_{L}$ being the Lagrangian covariance along the neutral particle trajectory (the fluctuations are assumed to be stationary, but not statistically homogeneous). For numerical evaluations of Eq. (27), $k$ must be such that $\left|\mathbf{r}-\mathbf{r}_{w}\right| / k$ (resp. $\left.\left|\mathbf{r}-\mathbf{r}_{w}\right| /\left(k \mathrm{v}_{0}\right)\right)$ be small compared to the spatial correlation length (resp. time) of fluctuations. Combining Eqs. (25)-(27) with the previous equation leads to the following important result:

$$
\left[\partial_{t}+\mathrm{v}_{0} \boldsymbol{\Omega} \cdot \nabla+\varpi^{S}\left(\left|\mathbf{r}-\mathbf{r}_{w}\right|\right)\right]\left\langle f^{S}\left(t, \mathbf{r}, \mathrm{v}_{0}, \mathbf{\Omega}\right)\right\rangle=0 .
$$

In other words, for the MGD, we have obtained the following exact statistical closure:

$$
\left\langle\tilde{\nu} \tilde{f}^{S}\right\rangle=\left(\varpi^{S}-\langle\nu\rangle\right)\left\langle f^{S}\right\rangle .
$$

The influx at the wall is given by $\Gamma_{0}=\Gamma_{p}=\mathrm{v}_{0}\left\langle f^{s}(0)\right\rangle$ (assuming that the recycling coefficient is unity). Equation (25) shows that the average neutral density can be obtained by solving a Boltzmann equation where the ionization rate is given by $\varpi^{S}$. In other words, fluctuations can be accounted for in transport codes upon replacing the ionization rate by a coarse-grained ionization rate (i.e., technically speaking, fluctuations renormalize the ionization rate) so that the average flux and sources can now be calculated with much less computational effort than what was necessary in our previous works. This result therefore opens the way for coupled plasma/neutral calculations, including the effects of turbulent fluctuations on neutrals. The average particle, momentum, and energy sources are given by

$$
\begin{gathered}
\left\langle S_{n}(\mathbf{r}, t)\right\rangle=\left\{\varpi^{S}\left(\mathbf{r}, t, \mathbf{v}_{0}, \boldsymbol{\Omega}\right)\left\langle f^{S}\left(\mathbf{r}, t, \mathbf{v}_{0}, \mathbf{\Omega}\right)\right\rangle\right\}, \\
\left\langle\mathbf{S}_{m}(\mathbf{r}, t)\right\rangle=m\left\{\mathbf{v}_{0} \varpi^{S}\left(\mathbf{r}, t, \mathbf{v}_{0}, \mathbf{\Omega}\right)\left\langle f^{S}\left(t, \mathbf{r}, \mathbf{v}_{0}, \mathbf{\Omega}\right)\right\rangle\right\},
\end{gathered}
$$


$\left\langle S_{E_{s}}(\mathbf{r}, t)\right\rangle=\left\{\left[\frac{1}{2} m_{s} \mathbf{v}_{0}^{2}-E_{s}^{l}\right] \varpi^{S}\left(\mathbf{r}, t, \mathbf{v}_{0}, \mathbf{\Omega}\right)\left\langle f^{S}\left(t, \mathbf{r}, \mathbf{v}_{0}, \mathbf{\Omega}\right)\right\rangle\right\}$,

where the curly brackets $\{\cdot\}$ stand for an average over the initial velocity $\mathbf{v}_{0}=\mathrm{v}_{0} \boldsymbol{\Omega}$. Equations (31)-(33) show that the effects of fluctuations are similar on all the source terms so that in the following we shall focus on the particle source.

As in Refs. 22 and 23, we introduce the parameter $a=\lambda_{L} / \ell$, where $\ell=\mathrm{v}_{0} /\langle\nu\rangle$ is the neutral particle mean free path and $\lambda_{L}$ the Lagrangian correlation length. The importance of time dependent effects can be measured by defining a correction factor $\kappa$, such that $a=\kappa a_{\text {adia }}$, where $a_{\text {adia }}=\lambda / \ell$ is the value taken by the parameter $a$ in the adiabatic approximation. For the covariance of Eq. (15), we get from Eq. (19)

$$
\kappa=\frac{\frac{\mathrm{v}_{0} \tau_{d}}{\lambda}}{1+\frac{\tau_{d}}{\lambda}\left|\left(\mathbf{v}_{0}-\mathbf{v}_{s}\right) \cdot \mathbf{\Omega}\right|},
$$

where $\lambda_{x}=\lambda_{y}=\lambda$. If $\mathbf{v}_{s}$ is zero, $\kappa \leq 1$ and time dependent effects have to be taken into account whenever $\tau_{d} \mathrm{v}_{0} / \lambda \lesssim 1$, where we recall that $\tau_{d}$ (resp. $\lambda$ ) represents the typical lifetime (resp. size) of turbulent structures. If the structures disappear faster than the neutral particle can fly through, time dependent effects play a role. If $\tau_{d} \mathrm{v}_{0} / \lambda \ll 1, a \simeq \mathrm{v}_{0} \tau_{d} / \ell$ $\ll a_{\text {adia. }}$. Now, for non-zero $\mathbf{v}_{s}$, the same reasoning can be made in the structure's rest frame, where the velocity of the neutral particle is $\mathbf{v}_{0}-\mathbf{v}_{s}$. In this case, for slowly decaying structures (i.e., large $\left.\tau_{d}\right), \kappa \simeq \mathrm{v}_{0} /\left|\left(\mathbf{v}_{0}-\mathbf{v}_{s}\right) \cdot \boldsymbol{\Omega}\right|$ and $a=\lambda_{\text {eff }} / \ell$ with $\lambda_{\text {eff }}=\lambda \mathrm{v}_{0} /\left|\left(\mathbf{v}_{0}-\mathbf{v}_{s}\right) \cdot \boldsymbol{\Omega}\right|$. In other words, neutrals see structures with an effective size, smaller than their actual size if $\mathbf{v}_{s} \cdot \mathbf{v}_{0}<0$. The correction factor $\kappa$ defined by Eq. (34) is plotted on Fig. 2(a) as a function of two independent dimensionless parameters, namely $\mathrm{v}_{0} \tau_{d} / \lambda$ and $\mathbf{v}_{s} \cdot \mathbf{\Omega} / \mathrm{v}_{0}$. Here, negative values of $\mathrm{v}_{s} / \mathrm{v}_{0}$ correspond to structures
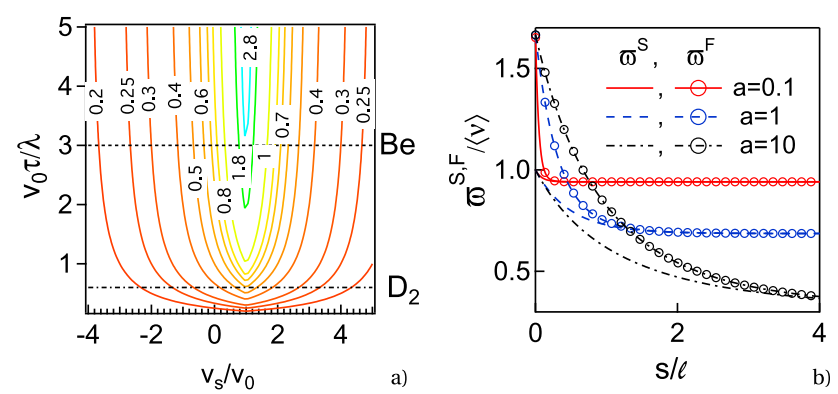

FIG. 2. (a) Contour plot of the correction factor $\kappa$ accounting for time dependent effects (Eq. (34)), as a function of $\tau_{d} v_{0} / \lambda$ and $v_{S} / v_{0}$. Negative values of $v_{S} / v_{0}$ correspond to structures moving opposite to neutrals. The increase of $\kappa$ for $\mathrm{v}_{S} / \mathrm{v}_{0} \simeq 1$ corresponds to the case where structures move at the same speed as neutrals. The two horizontal lines correspond to the values used in Sec. III C for $\mathrm{D}_{2}$ molecules and beryllium atoms. (b) Comparison between the effective ionization rates for the slow and fast recycling cases $\varpi^{S}$ from Eq. (27) or Eq. (36) (solid red line, $\mathrm{a}=0.1$; dashed blue line, $\mathrm{a}=1$; and dashed-dotted black line, $\mathrm{a}=10$ ), and $\varpi^{F}$ from Eq. (38) or Eq. (39) (same legend, with circles) as a function of the distance to the source $s$ in units of the average mean free path $\ell$, for $R=82 \%(\beta=1.5)$. Both rates are normalized to the average value $\langle\nu\rangle$. The fact that $\varpi^{F}>\varpi^{S}$ close to the source results from reionization in the structures responsible for recycling. In practice, the limiting value $\varpi_{\infty}(a, \beta)$ given by Eq. (35), identical for $\varpi^{S}$ and $\varpi^{F}$, is reached to a very good approximation at $s / \ell \simeq 2 a$. moving in the direction opposite to that of neutral particles (the most likely situation). For $\mathrm{v}_{s} / \mathrm{v}_{0} \simeq 1, \kappa$ increases markedly and takes values larger than unity, because neutrals are moving with the same velocity as the structures (for $\tau_{d} \rightarrow+\infty$, we then have $\kappa \rightarrow+\infty$ : Everything happens as if the plasma was spatially homogenous). The effective ionization rate $\varpi_{s}$ given by Eq. (27) is plotted as a function of the distance to the source normalized to the average neutral mean free path $\ell$ on Fig. 2(b), for the covariance Eq. (15) (in the isotropic case), and for different values of the parameter $a$. It is smaller than the ionization rate calculated for the average plasma parameters so that neutrals will penetrate deeper in the plasma in presence of turbulence, even more so when the Lagrangian correlation length becomes larger than the neutral mean free path. Physically, this behavior stems from the fact that most of the neutrals which penetrate deeply do so in realizations for which the density along their trajectory was low. The larger the correlation length, the longer the atom will experience this low density, and the farther it is likely to go. If the fluctuations are statistically homogeneous, for large $\left|\mathbf{r}-\mathbf{r}_{w}\right|$, in practice after a distance of a few correlation lengths $\lambda$ from the source, $\varpi^{S}\left(\left|\mathbf{r}-\mathbf{r}_{w}\right|\right) \rightarrow \varpi_{\infty}$, where the value of $\varpi_{\infty}$ follows from Szegö's theorem on Toeplitz matrices. ${ }^{22,42}$ For the covariance given by Eq. (15)

$$
\varpi_{\infty}=\langle\nu\rangle \frac{g-1}{2 R K},
$$

where $K=\left(\sigma_{n_{e}} / \mathrm{v}_{0}\right) \lambda_{L}=R \lambda_{L} / \ell$ is the Kubo number, ${ }^{43,44}$ and $g=\sqrt{1+4 R K}$. For other homogeneous covariances, $\varpi_{\infty}$ can be calculated numerically (see Appendix B of Ref. 22). Let us emphasize that Eq. (35) is fully consistent with the asymptotic behavior of $f^{S}=\frac{\left\langle\Gamma_{0}\right\rangle}{\mathrm{v}_{0}} Z_{e}\left(u_{c}\right)$, where $Z_{e}$ is given by Eq. (18) and $u_{c}=\overline{\sigma_{i o} \mathrm{~V}_{e}} / \mathrm{v}_{0}$. It is then straightforward to show (see Appendix B) that

$$
\varpi^{S}(s)=\frac{\mathrm{v}_{0}}{2 R^{2} \lambda_{L}}\left[g \frac{\frac{g^{2}+1}{2 g}+\tanh \left(\frac{g s}{2 \lambda_{L}}\right)}{1+\frac{g^{2}+1}{2 g} \tanh \left(\frac{g s}{2 \lambda_{L}}\right)}-1\right],
$$

with $s=\left|\mathbf{r}-\mathbf{r}_{w}\right|$. The latter result is an exact reformulation of Eq. (27) in terms of elementary functions, valid for an exponential covariance. Note that Eq. (35) follows from Eq. (36) in the limit $g s / \lambda_{L} \gg 1$.

\section{B. Fast recycling}

The particle flux at the wall $\Gamma_{p}$ is now assumed to be related to the fluctuating plasma density through $\Gamma_{p}=n_{e} \mathrm{v}_{\text {eff }}$ (hence $\left.f\left(t, \mathbf{r}, \boldsymbol{\Omega}, \mathrm{v}_{0}\right) \propto n_{e}\left(\mathbf{r}_{w}, t-\mid \mathbf{r}-\mathbf{r}\right) \mid / \mathrm{v}_{0}\right)$ according to Eq. (3)), where $v_{\text {eff }}$ is constant. The average flux is then $\left\langle\Gamma_{p}\right\rangle=\left\langle n_{e}\right\rangle \mathrm{v}_{\text {eff }}$ so that $\mathrm{v}_{\mathrm{eff}}$ can be identified as the effective convection velocity introduced in, e.g., Refs. 12 and 45. The average in Eq. (25) is given by

$$
\begin{aligned}
\left\langle\nu(\mathbf{r}, t) f^{F}(\mathbf{r}, t)\right\rangle & =\left.\frac{\overline{\sigma_{i o} \mathbf{v}_{e}} \mathrm{v}_{e f f}}{\mathrm{v}_{0}} \frac{\delta^{2} Z[u]}{\delta u\left(\left|\mathbf{r}-\mathbf{r}_{w}\right|\right) \delta u(0)}\right|_{u=u_{c}} \\
& =\varpi^{F}\left(\left|\mathbf{r}-\mathbf{r}_{w}\right|\right)\left\langle f^{F}\right\rangle,
\end{aligned}
$$


where $u_{c}=\overline{\sigma_{i o} \mathrm{v}_{e}} \mathrm{v}_{0}$, and where the effective ionization rate $\varpi^{F}\left(\left|\mathbf{r}-\mathbf{r}_{w}\right|\right)$ is defined by (see Appendix D)

$$
\begin{aligned}
\varpi^{F}\left(\left|\mathbf{r}-\mathbf{r}_{w}\right|\right)= & \varpi^{S}\left(\left|\mathbf{r}-\mathbf{r}_{w}\right|\right)+\frac{\mathbf{v}_{0}}{\left|\mathbf{r}-\mathbf{r}_{w}\right|} \lim _{k \rightarrow+\infty} k\left(A^{-1}\right)_{k k} \\
& \times \frac{\left(A^{-1}\right)_{11}-\left(B^{-1}\right)_{11}}{1-\left(A^{-1}\right)_{11}}
\end{aligned}
$$

where $\mathbf{B}$ is a $k-1 \times k-1$ matrix with entries $B_{i j}=A_{i j}$. In statistically homogeneous cases, the diagonal elements of $\mathbf{A}$ are all equal so that Eq. (38) reduces to Eq. (9) of Ref. 19. As a result of Eq. (37), the coarse-grained transport equations are of the same form in the slow and fast recycling cases, but the effective rates are different. The sources of particles, momentum, and energy are given by Eqs. (31)(33), using $\varpi^{F}$ and $\left\langle f^{F}\right\rangle$ instead of $\varpi^{S}$ and $\left\langle f^{S}\right\rangle$. The rate $\varpi^{F}$ is plotted on Fig. 2(b) for different values of the parameter $a$ and the same correlation function used for $\varpi^{S}$ on Fig. 2(a). It is clear that $\varpi^{F} \geq \varpi^{S}$, with $\varpi^{F}>\varpi^{S}$ close to the wall $\left(\varpi^{F} / \varpi^{S}=1+1 / \beta\right.$ for $\left.\mathbf{r}=\mathbf{r}_{w}\right)$. In fact, in the fast recycling case the source is large only in regions where the density is high. Therefore, reionization is much stronger close to the wall in the fast recycling model. However, for large $s=\left|\mathbf{r}-\mathbf{r}_{w}\right|$, we have $\varpi^{F}(s) \sim \varpi^{S}(s)$. In practice, as shown of Fig. 2, this asymptotic regime is reached for $s$ larger than a few correlation lengths $\lambda$, since then the density fluctuations at location $s$ are mostly uncorrelated to those at the wall. For the exponential covariance of Eq. (15), $\varpi^{F}$ as defined by Eq. (38) can be expressed in terms of elementary functions. We have (see Appendix B)

$$
\begin{aligned}
\varpi^{F}(s)= & \varpi^{S}(s) \\
& +\frac{\mathrm{v}_{0}}{2 \lambda_{L}} \frac{g^{2}\left(g^{2}-1\right)\left(1-\tanh ^{2}\left(\frac{g s}{2 \lambda}\right)\right)}{\left(g+\tanh \left(\frac{g s}{2 \lambda_{L}}\right)\right)\left(2 g+\left(g^{2}+1\right) \tanh \left(\frac{g s}{2 \lambda_{L}}\right)\right)},
\end{aligned}
$$

with $\varpi^{S}$ given by Eq. (36). The asymptotic result of Eq. (35) is recovered in the limit $g s / \lambda_{L} \gg 1$.

\section{Application: The effects of structure propagation}

Here, we focus on the role of structure propagation. We consider two cases, namely $\mathrm{D}_{2}$ molecules with $E_{0}=0.03 \mathrm{eV}$ (corresponding to thermal release from the wall) and beryllium atoms such that $E_{0}=1.7 \mathrm{eV}$ (half of the surface binding energy, which corresponds to the maximum of the Thompson distribution ${ }^{46}$ ). We set $\lambda_{x}=\lambda_{y}=2 \mathrm{~cm}, \tau_{d}$ $=10 \mu \mathrm{s}$, and $\mathrm{v}_{s x}=0,-1$ and $-10 \mathrm{~km} / \mathrm{s}$ (here the $\mathrm{x}$ axis represents the radial direction). This corresponds to coherent structures moving towards the wall, i.e., in a direction opposite to that of recycling neutrals in the radial direction. $\mathrm{v}_{s x}=0$ corresponds to the case investigated in previous works, ${ }^{19,22,23}$ and $\mathrm{v}_{s x}=-1 \mathrm{~km} / \mathrm{s}$ is a realistic value. ${ }^{9}$ For $\mathrm{D}_{2}$ molecules we have $\tau_{d} \mathrm{v}_{0} / \lambda_{x}=0.6$, while for $\mathrm{Be}$ atoms $\tau_{d} \mathrm{v}_{0} / \lambda_{x}=3$ (the corresponding horizontal lines are plotted on Fig. 2(a)). Tables I and II show the values of $\mathrm{v}_{s x} / \mathrm{v}_{0}$ and $\kappa$ for the three values of $\mathrm{v}_{s x}$ considered here. The overall
TABLE I. Values of $\mathrm{v}_{s x} / \mathrm{v}_{0}$ and of the correction factor accounting for time dependent effects, for the parameters given in the text and $\mathrm{v}_{s x}=0,-1$, and $-10 \mathrm{~km} / \mathrm{s}$, for $\mathrm{D}_{2}$ molecules.

\begin{tabular}{lccc}
\hline \hline $\mathrm{v}_{s x} / \mathrm{v}_{0}$ & 0 & -0.8 & -8 \\
$\kappa\left(\mathrm{D}_{2}\right)$ & 0.4 & 0.3 & 0.1 \\
\hline \hline
\end{tabular}

importance of the effect of fluctuations depends on the value of $a=\kappa a_{\text {adia }}$, where $a_{\text {adia }}=\lambda_{x} / \ell$, with the average mean free path $\ell$ given by $\ell=v_{0} /\langle\nu\rangle$. Three loss channels are considered for $\mathrm{D}_{2}$, (processes 2.2.5, 2.2.9, and 2.2.10 in the HYDHEL database $^{47}$ ), namely

$$
\begin{gathered}
D_{2}+e^{-} \rightarrow D(1 s)+D(1 s)+e^{-}, \\
D_{2}+e^{-} \rightarrow D_{2}^{+}+e^{-}+e^{-}, \\
D_{2}+e^{-} \rightarrow D^{+}+D(1 s)+e^{-}+e^{-} .
\end{gathered}
$$

This leads to $\left\langle\nu_{D 2}\right\rangle \simeq 2.4 \times 10^{5} \mathrm{~s}^{-1}$ for $n_{e}=5 \times 10^{18} \mathrm{~m}^{-3}$, and $T_{e}=50 \mathrm{eV}$ so that $a_{\text {adia }} \simeq 4$. For beryllium, we rely on the ADAS database ${ }^{48}$ (scd93 datas), yielding $\left\langle\nu_{\mathrm{Be}}\right\rangle \simeq 6.5$ $\times 10^{5} \mathrm{~s}^{-1}$ for the ionization rate in the same plasma conditions. Thus, $a_{\text {adia }} \simeq 2$.

In the following, we present radial density profiles for the conditions considered above, obtained in a $2 \mathrm{D}$ slab geometry with the coarse-grained transport model for neutrals implemented in the EIRENE Monte Carlo code. The main lines of the technical implementation have been described in Ref. 19. Here, we discuss the case where several destruction processes exist for a given species, as it is the case for $\mathrm{D}_{2}$. It is easily seen from formulas (36) and (39) that the coarsegrained ionization rate is not additive, in the sense that if $\nu=\sum \nu_{i}, \varpi \neq \sum \varpi_{i}$, where $\varpi$ is calculated using the total rate $\nu$. In the numerical implementation, $\varpi$ must therefore be used when calculating the neutral particle free path. The probability of destruction channel $k$ is then obtained from the local rates, as in the original Monte Carlo procedures, namely $p_{k}=\nu_{k} / \sum \nu_{i}$, since all the rates are linear in density so that $p_{k}$ is independent on density. The correct expression for the electron source is then

$$
\left\langle S_{n}(\mathbf{r})\right\rangle=\left(\sum_{k} p_{k}(\mathbf{r}) \theta_{k}\right)\left\{\varpi_{S}\left(\left|\mathbf{r}-\mathbf{r}_{w}\right|\right)\left\langle f^{S}\left(\mathbf{r}, \mathrm{v}_{0}, \mathbf{\Omega}\right)\right\rangle\right\},
$$

where $\theta_{k}$ is the number of electrons created in channel $k$ ( $\theta=0$ for the process defined by Eq. (40), and $\theta=1$ for those represented by Eqs. (41) and (42)), and where the curly brackets $\{\cdot\}$ stand for the average over the neutral particle velocity $\mathbf{v}_{0}$. The results of the numerical calculations for $\mathrm{D}_{2}$ (resp. Be) are presented on Fig. 3 (resp. Fig. 4) in logarithmic

TABLE II. Values of $\mathrm{v}_{s x} / \mathrm{v}_{0}$ and of the correction factor accounting for time dependent effects, for the parameters given in the text and $\mathrm{v}_{s x}=0,-1$, and $-10 \mathrm{~km} / \mathrm{s}$, for Be atoms.

\begin{tabular}{lccc}
\hline \hline $\mathrm{v}_{s x} / \mathrm{v}_{0}$ & 0 & -0.17 & -1.7 \\
$\kappa(\mathrm{Be})$ & 0.75 & 0.7 & 0.3 \\
\hline \hline
\end{tabular}



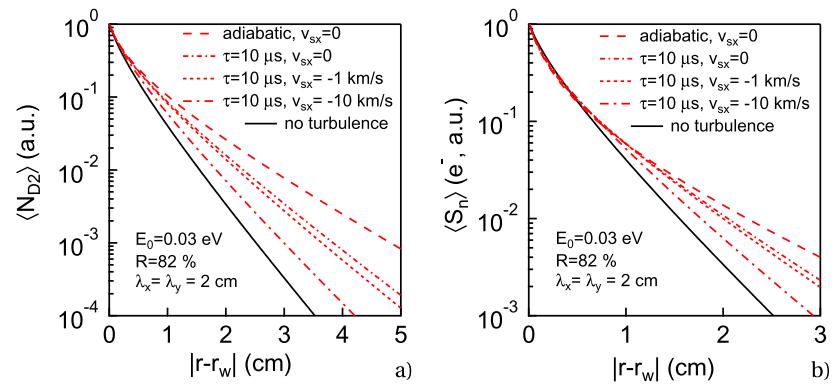

FIG. 3. (a) Average density and (b) average ionization source, for $D_{2}$ molecule in the slow recycling case, with $R=82 \%, \lambda_{x}=\lambda_{y}=2 \mathrm{~cm}, \tau_{d}=10 \mu \mathrm{s}$ and $\mathrm{v}_{s x}=0$ (dashed-dotted), -1 (dotted), $-10 \mathrm{~km} / \mathrm{s}$ (long dashed-dotted), $E_{0}=0.03 \mathrm{eV}$. The adiabatic approximation for $\mathrm{v}_{s}=0$ yields the red dashed curve. The difference between the latter and three former profiles gives the magnitude of time dependent effects, which play an important role for these conditions. The effect of structure propagation for realistic velocities is rather weak here.

scale, assuming a constant neutral velocity $\mathbf{v}_{0}$ with a cosine angular distribution. The slow recycling model is used for molecules, assuming that the thermal release mechanism is dominant, while the fast recycling model describes well the sputtered flux of beryllium atoms. The turbulence free radial profiles are the solid black curves on all figures, and the red dashed curve is the adiabatic case. The short dashed-dotted curve, the dotted curve, and the dashed-dotted curve correspond to the time dependent case with, respectively, $\mathrm{v}_{s x}=0$, -1 , and $-10 \mathrm{~km} / \mathrm{s}$. The average particle source is less affected by fluctuations than the average density in the slow recycling case, as discussed in Refs. 22 and 23. The situation is reversed in the fast recycling case. In accordance with the numbers in Tables I and II, time dependent effects are markedly weaker for beryllium atoms than for $D_{2}$, essentially because their velocity is larger. For $\mathrm{D}_{2}$ molecules, the effects of structure propagation are rather weak for $\mathrm{v}_{s x}=1 \mathrm{~km} / \mathrm{s}$, but become very significant for structures 10 times faster (a situation that does not seem realistic).

\section{SENSITIVITY TO THE CHOICE OF STATISTICAL DESCRIPTION OF THE FLUCTUATIONS}

Our stochastic model for density fluctuations is based on the observation that the PDF of density fluctuations at one point is well represented by a Gamma distribution. We then assume that the multivariate statistical properties of density fluctuations are well described by the MGD, and use the 2-point covariance function to fully specify the MGD. In this section, we use the result obtained for the cumulants of the optical depth in Sec. II D, namely Eq. (21), to investigate the sensitivity of our results to the higher order (i.e., r-points, with $r>2$ ) statistical properties of the fluctuations. Then, we discuss the case where the PDF is Log-normal, the latter also providing a reasonable description of density fluctuations in the far SOL of TCV, ${ }^{24}$ as well as in RFX. ${ }^{49}$

\section{A. Role of the $\mathrm{N}$-point correlation functions}

The cumulant expansion theorem ${ }^{50,51}$ yields the following expression for the characteristic function of the optical depth:
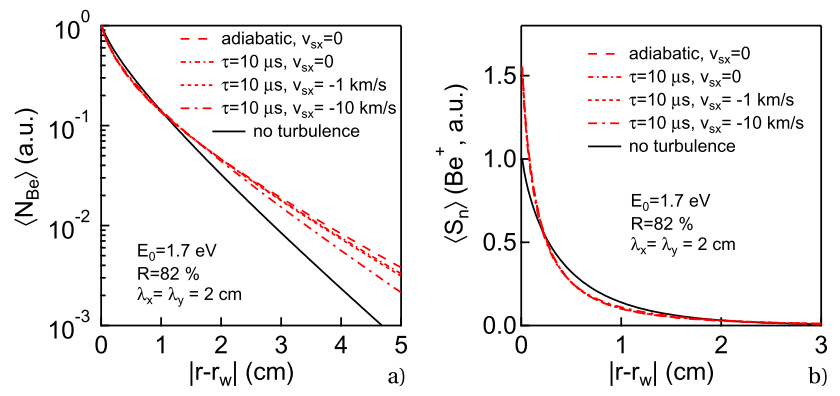

FIG. 4. (a) Average density and (b) particle source for beryllium atoms in the fast recycling model, with $E_{0}=1.7 \mathrm{eV}$. The curves are labelled the same way as in Fig. 4. Time dependent effects are much weaker here than for $\mathrm{D}_{2}$ molecules.

$$
\left\langle e^{-u \tau}\right\rangle=\exp \left(\sum_{r=1}^{\infty}(-1)^{r} \frac{\left\langle\left\langle\tau(s, \boldsymbol{\Omega})^{r}\right\rangle\right\rangle}{r !} u^{r}\right)
$$

which can also be obtained upon expanding the cumulant generating function in its Taylor series, see Eq. (20). Equation (44) can in principle be used in conjunction with Eq. (21) to calculate a numerical estimate of $\left\langle f^{S}\right\rangle$ by taking $u=1$ and truncating the series to a finite order. Equation (44) thus provides a starting point to investigate the sensitivity of the results to changes in the r-point covariances for $r>2$, keeping the marginal distribution unchanged. However, two technical difficulties appear when doing so. First, as shown in Appendix $\mathrm{C}$, the cumulant expansion converges only if the largest eigenvalue $e_{\max }(s)$ of the covariance operator is such that $e_{\max }(s)<1 / 2$. For a given value of $s, e_{\max }$ is obtained from the solution of a transcendental equation (see Eqs. (67) and (68)), following standard theory of Fredholm integral equations. This point is clearly illustrated in the limit $s / \lambda_{L} \ll 1$, where the series can be resummed

$$
-\beta \sum_{r=1}^{\infty} \frac{(-1)^{r+1}}{r}\left(\frac{s u}{\ell \beta}\right)^{r}=-\beta \ln \left(1+\frac{s u}{\ell \beta}\right),
$$

provided $s /(\ell \beta)<1 \quad(u=1$ here $)$. The latter condition is precisely that obtained by imposing $e_{\max }(s)<1 / 2$, since for a given $s$ and as $\lambda_{L} \rightarrow+\infty$, Eq. (67) reduces to $\omega_{1} s \simeq 1 /\left(\omega_{1} \lambda_{L}\right)$ (where $\omega_{1}$ is the smallest root) so that $e_{\max }=s /(2 \beta \ell)$. Note that the solution given by Eq. (45) is nevertheless valid for all positive values of $s$, as can be seen from comparison to Eq. (A5) of Ref. 22. The fact that the cumulant expansion has a finite convergence radius is related to the choice of the Gamma statistics (in other words, the cumulant expansion of the Gamma distribution has a finite convergence radius) and manifests itself in other works dealing with stochastic modeling of plasma density fluctuations (see Eqs. (24) and (25) of Ref. 26). A more difficult issue has to do with the mathematical consistency of the modified stochastic process (technically, its realizability ${ }^{32}$ ). In other words, it is not clear that modifying r-point covariances lead to a proper stochastic process. In the following, we will distinguish between two levels of realizability. The first one is to ensure realizability for the optical depth $\tau$, in the sense that its PDF takes only positive value (i.e., its characteristic 
function is positive definite). The second one is more demanding and requires that the modified underlying multivariate statistics be realizable (that is, the multivariate PDF takes only positive values). This question will not be addressed here.

Now, we look for a modification of the cumulants such that Eq. (44) can be resummed and that both the 1-point marginal of $n_{e}$ and its 2-point covariance are unchanged. This can be achieved upon replacing the integral in the r.h.s. of Eq. (21) by

$$
\begin{aligned}
& \int_{0}^{s} d s_{1} \ldots \int_{0}^{s} d s_{r}\left(C_{12} G_{23} \ldots C_{r 1}\right)^{1 / 2} \\
& \quad \simeq \int_{0}^{s} d s_{1} \ldots \int_{0}^{s} d s_{r}\left(C_{r 1}\right)^{1 / 2}=\frac{\left\langle n_{e}\right\rangle \overline{\sigma_{i o} \mathbf{v}_{e}}}{\sqrt{\beta}} \mathcal{J}(s) s^{r-2} .
\end{aligned}
$$

Here, we have defined

$$
\mathcal{J}(s)=\int_{0}^{s} d s_{1} \int_{0}^{s} d s_{r}\left(\zeta\left(s_{r}-s_{1}\right)\right)^{1 / 2}
$$

where $\zeta$ is the correlation function introduced in Eq. (14). For the exponential covariance defined by Eq. (15),

$$
\mathcal{J}(s)=4 \lambda_{L}^{2}\left(\frac{s}{\lambda_{L}}+2\left(e^{-s / 2 \lambda_{L}}-1\right)\right) .
$$

The series in Eq. (44) is then given by

$$
\begin{aligned}
& -\frac{s}{\ell} u+\beta \frac{\mathcal{J}(s)}{s^{2}} \sum_{r=2}^{+\infty} \frac{(-1)^{r}}{r}\left(\frac{s u}{\ell \beta}\right)^{r} \\
& =-\frac{s}{\ell} u+\frac{\mathcal{J}(s)}{\ell s} u-\beta \frac{\mathcal{J}(s)}{s^{2}} \ln \left(1+\frac{s u}{\ell \beta}\right) .
\end{aligned}
$$

Again, this result only holds for $s /(\ell \beta) \leq 1$, for otherwise the series on the 1.h.s. is not convergent. This time, we will assume that the resummed result is correct for larger values of $s$, as it turned out to be the case for infinite correlation lengths, see Eq. (45). The final result for the characteristic function is

$Z_{c}(u)=\left\langle e^{-u \tau}\right\rangle=\frac{\Gamma_{0}}{\mathrm{v}_{0}}\left(1+\frac{s u}{\ell \beta}\right)^{-\beta \mathcal{J}(s) / s^{2}} \exp \left[-\frac{s-\mathcal{J}(s) / s}{\ell} u\right]$.

The latter function is nothing but the Fourier transform of a Gamma distribution with displacement $\langle\nu\rangle(s-\mathcal{J}(s) / s)$, shape parameter $\beta \mathcal{J}(s) / s^{2}$, and scale parameter $\langle\nu\rangle s$. In other words, the PDF of the optical depth can be calculated exactly in this case, and is found to be realizable (it takes positive values only and is thus a proper PDF). For $s \ll \lambda_{L}$, one has $\tau \simeq \nu s / \mathrm{v}_{0}$ so that the PDF of the optical depth becomes a Gamma distribution with zero displacement, shape parameter $\beta$, and scale parameter $\langle\nu\rangle / \beta$. This means that the 1-point marginal of $\nu$ is indeed unchanged. One could also start in reverse order, imposing the optical depth PDF, from which Eq. (50) is obtained by Laplace transform, and calculating the cumulants from the derivatives of $\psi(u)=\ln \left\langle e^{-u \tau}\right\rangle$. This circumvents the problem of the divergence of the cumulant expansion for $s /(\ell \beta)>1$. The neutral distribution $\left\langle f_{c}^{S}\right\rangle$ is given by $\left\langle f_{c}^{S}\right\rangle=\Gamma_{0} / \mathrm{v}_{0} Z_{c}$ $(u=1)$. The limits defined by Eqs. (A5) and (A4) of Ref. 22 are recovered from Eq. (50), upon noting that $\mathcal{J}(s)=s^{2}$ for $\lambda \rightarrow \infty$ and $\mathcal{J}(s) \rightarrow 0$ for $\lambda \rightarrow 0$. The modified cumulants of order two to four are compared on Fig. 5 to their counterpart for the MGD, given by Eqs. (22)-(24), for $R=82 \%$ (i.e., $\beta=1.5$ ), and the covariance of Eq. (15) for $a=\lambda_{L} / \ell$ $=1$. The differences are quite marked for large values of $s$. Note that the second cumulants of the optical depth differ, even though the covariance of the density field is unchanged. Fig. 5(b) compares $\left\langle f_{c}^{S}\right\rangle$ (black solid lines) to the exact result obtained from Eq. (18) (dashed red curves), with four different values of the ratio of the Lagrangian correlation length $\lambda_{L}$ to the neutral mean path $a=\lambda_{L} / \ell=0.1,1,10$, and 100 . The two results are in good agreement on the range of $s$ plotted here. Except for $a \sim 1$, the discrepancies are of the order of a few percents. The largest differences occur for intermediate values of $a$ and large $s$. These results are encouraging, since they suggest that as far as the average properties of the transport of neutral particles are concerned, the PDF of the density field at one point and the two point covariance function provides enough information to get reliable results.

\section{B. Log-normal vs Gamma PDF}

We now consider the case where the PDF is Log-normal instead of Gamma. The Log-normal distribution has a natural multivariate extension, which has been used recently in studies of radiation transport in stochastic media. ${ }^{52}$ The Gamma and Log-normal marginal PDFs are compared on Fig. 6(a), with the same average density $\left\langle n_{e}\right\rangle$ and the same relative fluctuation level $R=82 \%$, which corresponds to $\beta=1.5$. The resulting neutral density profiles, in the adiabatic case with $\lambda \rightarrow+\infty$, and assuming slow recycling, are plotted on Fig. 6(b). Fluctuations enhance the penetration of neutrals in both cases, although the effects are slightly less pronounced for the Log-normal (red dashed curve) than for the Gamma distribution. This is consistent with the observation made on Fig. 6(a) that the probability of having low density realizations $\left(n_{e} /\left\langle n_{e}\right\rangle<0.5\right)$ is much smaller for the Log-normal than for the Gamma distribution. We conclude from this comparison that the general conclusions we have drawn on
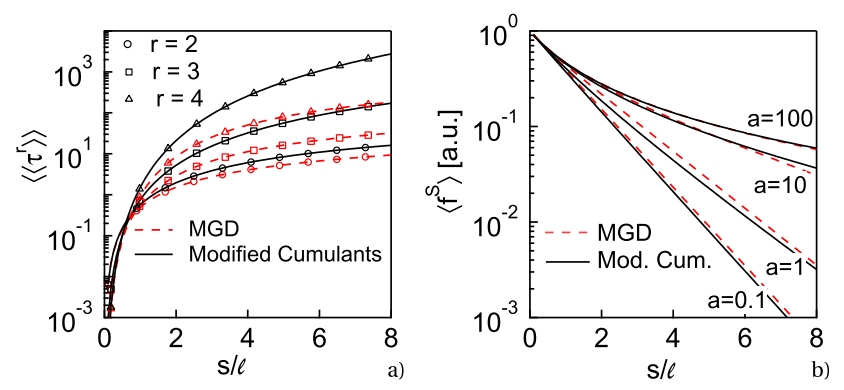

FIG. 5. (a) Comparison between the exact and modified cumulants of order 2 to 4 as function of $s=\left|\mathbf{r}-\mathbf{r}_{w}\right|$, for $R=82 \%(\beta=1.5)$ and the exponential covariance of Eq. (15) with $a=\lambda_{L} / \ell=1$. (b) Comparison between the average neutral density with modified statistics $\left\langle f_{c}^{S}\right\rangle$ (black solid lines) and the exact result for the MGD (dashed red lines) as a function of $\mathrm{s}$, for the same parameters as in (a), but with $a=0.1,1,10$, and 100. The discrepancies are small, given the important differences in the cumulants. 

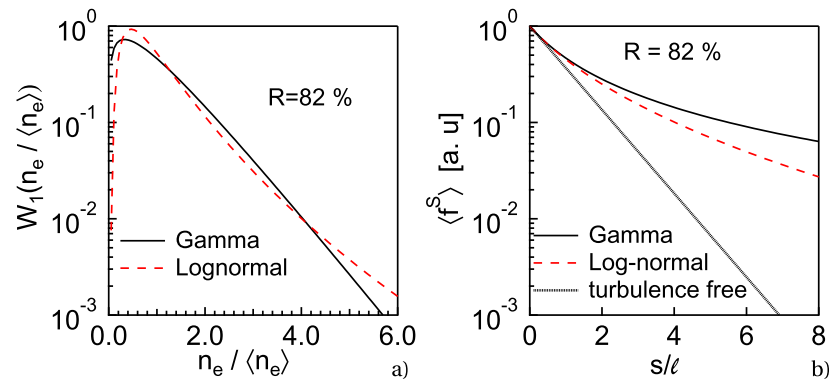

FIG. 6. (a) Comparison between the Gamma distribution (black solid line) and the Log-normal distribution (red dashed line), for $R=82 \%$. The Lognormal distribution has a heavier tail than the Gamma, and leads to smaller probabilities for low density realizations $\left(n_{e} /\left\langle n_{e}\right\rangle<0.5\right)$. (b) Comparison between the average neutral density in the case of Gamma statistics (black solid line) and Log-normal statistics (red dashed line), in the adiabatic case and with $\lambda \rightarrow \infty$. The effects are qualitatively the same but slightly less pronounced in the Log-normal case.

the effects of fluctuations on the transport of neutral particles are also valid for Log-normal statistics. Now, the interesting question is whether a simple coarse-grained transport model can be derived in the same way as we did for the MGD. Our starting point was the characteristic function $Z[\mathbf{u}]$ of the MGD, given by Eq. (12). Perhaps surprisingly, the characteristic function of the Log-normal distribution does not have an expression in terms of elementary functions. However, in the univariate case, it can be shown to obey a functional equation. ${ }^{53}$ From this result, it is straightforward to show that in the slow recycling case we have

$$
\mathrm{v}_{0} \boldsymbol{\Omega} \cdot \nabla\left\langle f^{S}(\mathbf{r})\right\rangle=-\langle\nu\rangle\left\langle f^{S}\left(\mathbf{r}+R^{2}\left|\mathbf{r}-\mathbf{r}_{w}\right| \boldsymbol{\Omega}\right)\right\rangle,
$$

where $R$ is the relative fluctuation level of the density. In other words, after averaging over fluctuations, the neutral loss rate at point $\mathbf{r}$ is proportional to the neutral density at a point further away from the source, namely $\mathbf{r}^{\prime}=\mathbf{r}+R^{2}\left|\mathbf{r}-\mathbf{r}_{w}\right| \boldsymbol{\Omega}$. For $R=82 \%,\left|\mathbf{r}^{\prime}-\mathbf{r}_{w}\right| \simeq 1.67\left|\mathbf{r}-\mathbf{r}_{w}\right|$. Therefore, in the Log-normal case, the effect of fluctuations does not simply amount to introduce a coarse-grained ionization rate (technically, Eq. (51) is a differential functional equation of the advanced type, e.g., Ref. 54). These results show that even though assuming Log-normal instead of Gamma statistics does not strongly modify the average neutral density, the choice of the Gamma distribution greatly facilitates calculations, since a simple coarse-grained transport model is available. For the multivariate Log-normal case, the only available option at the present time is to rely on sampling methods, that is, solving the Boltzmann equation for a large number of realizations of the density field. This approach can either rely on brute force, by Cholesky decomposition of the covariance matrix, ${ }^{2,23}$ or on more sophisticated methods, e.g., Karhunen-Loève decomposition for the plasma density combined to stochastic collocation for the density of neutrals (the covariance of which is not known a priori). ${ }^{52}$

\section{CONCLUSION}

The effects of turbulent fluctuations on neutral particles transport in the edge plasma of magnetic fusion devices have been investigated. We have focused on the development of coarse-grained transport models embedding fluctuations into the kinetic transport equation itself. Our previous work on this subject showed that the effects of fluctuations would be much weaker on fuel atoms than on molecules, because the mean free path $\ell$ of the former is significantly larger than the size of the turbulent structures (measured by the correlation length $\lambda$ ). Indeed, in the adiabatic limit where fluctuations are frozen in time, the effects of fluctuations increase with the parameter $a=\lambda / \ell$ (for a given relative fluctuation level and wall response model). As a result, we have concentrated our efforts on the scattering free case, which provides a good description of the transport of impurity atoms and molecules in not too high plasma density regimes (e.g., main chamber recycling), when elastic proton-molecular collisions can be safely neglected. In the scattering free case, multivariate Gamma statistic for density fluctuations leads to a coarsegrained transport model, where the average particle source and the average density are related in a simple way. In fact, in the one speed transport problem the effect of fluctuations amounts to renormalize the ionization rate. This reformulation of the problem allows one to relax the adiabatic assumptions on which our previous works relied. The time correlations of the fluctuations, including the ballistic propagation of coherent turbulent structures, are now taken into account. Retaining these effects amounts to replace the turbulence correlation length $\lambda$ by the Lagrangian turbulence correlation length $\lambda_{L}$. If neutrals are propagating in the direction opposite to that of turbulent structures, as is the most likely situation for molecules and impurities, $\lambda_{L}<\lambda$ and the impact of fluctuations is reduced compared to the adiabatic case. However, the effects of fluctuations on neutral particle transport remain clearly substantial for realistic values of SOL parameters, and are more pronounced in high density $n_{e}>5 \times 10^{18} \mathrm{~m}^{-3}$ and high temperature $T_{e}>10 \mathrm{eV}$ conditions. The model has been implemented in the EIRENE Monte Carlo code, and future work will concentrate on the application of our model to ITER and DEMO cases.

\section{ACKNOWLEDGMENTS}

This work was carried out within the framework of the European Fusion Development Agreement and the French Research Federation for Fusion Studies. It is supported by the European Communities under the contract of Association between Euratom and FZJ, and Euratom and CEA. The views and opinions expressed herein do not necessarily reflect those of the European Commission. Financial support was also received from the Agence Nationale de la Recherche, project ANR-11-BS09-023 (SEDIBA).

\section{APPENDIX A: SUMMARY OF PARAMETER DEFINITIONS AND THEIR RELATIONS}

We summarize here relations between some parameters frequently used in this work. The average density for the PDF defined by Eq. (10) is $\left\langle n_{e}\left(\mathbf{r}_{i}\right)\right\rangle=\alpha_{i} \beta$, and the variance is $\sigma_{n_{e}}^{2}\left(\mathbf{r}_{i}\right)=\left\langle n_{e}^{2}\right\rangle-\left\langle n_{e}\right\rangle^{2}=\alpha_{i}^{2} \beta$. The relative fluctuation level $R$ is thus given by 


$$
R=\frac{\sigma_{n_{e}}}{\left\langle n_{e}\left(\mathbf{r}_{i}\right)\right\rangle}=\frac{1}{\sqrt{\beta}}=\sqrt{\frac{2}{M}} .
$$

The neutral particle mean free path $\ell$ is defined by

$$
\ell=\frac{\mathrm{v}_{0}}{\langle\nu\rangle}=\frac{\mathrm{v}_{0}}{\left\langle n_{e}\right\rangle \overline{\sigma_{i o} \mathrm{v}_{e}}},
$$

where $\overline{\sigma_{i o} \mathrm{~V}_{e}}$ is the rate coefficient. The turbulence correlation length along the direction $\boldsymbol{\Omega}$ is given by

$$
\lambda=\frac{1}{\sigma_{n_{e}}^{2}} \int_{0}^{+\infty} C(s \boldsymbol{\Omega}, 0) d s,
$$

while the Lagrangian correlation length is

$$
\lambda_{L}=\frac{1}{\sigma_{n_{e}}^{2}} \int_{0}^{+\infty} C\left(s \boldsymbol{\Omega},-\frac{s}{\mathrm{v}_{0}}\right) d s .
$$

In the adiabatic case, $\lambda_{L} \simeq \lambda$.

\section{APPENDIX B: THE GRENANDER-SZEGÖ FORMULA}

The determinant $D_{k}$ of the $k \times k$ matrix $\mathbf{A}$ with entries $A_{i j}=\delta_{i j}-\alpha^{-1} \rho^{|i-j|}$ is given, according to Ref. 34, by

$$
\begin{aligned}
D_{k}= & \frac{\rho^{k}}{1-\rho^{2}} \frac{1}{\sin \theta}(\sin ((k+1) \theta)-2 \rho \sin (k \theta) \\
& \left.+\rho^{2} \sin ((k-1) \theta)\right),
\end{aligned}
$$

where $\theta=\arccos \left(\left(1+\rho^{2}-\left(1-\rho^{2}\right) / \alpha\right) /(2 \rho)\right)$ (note that $\theta$ might take complex values). In our case, $\rho=\exp \left(-s /\left(2 \lambda_{L} k\right)\right)$, and $\alpha=-\beta k /\left(\left\langle n_{e}\right\rangle s u\right)$. We are interested in the limit of $D_{k}$ as $k$ tends to infinity. Some straightforward (but tedious) algebra leads to

$$
\lim _{k \rightarrow+\infty} D_{k}=\exp \left(-\frac{s}{2 \lambda_{L}}\right)\left[\cosh \left(\frac{g s}{2 \lambda_{L}}\right)+\left(\frac{g^{2}+1}{2 g}\right) \sinh \left(\frac{g s}{2 \lambda_{L}}\right)\right],
$$

with $g=\sqrt{1+4 \lambda_{L}\left\langle n_{e}\right\rangle u / \beta}$, from which Eq. (18) immediately follows. The easiest way to obtain Eq. (36) for $\varpi^{S}$ is then to differentiate Eq. (18) taken for $u=u_{c}=\overline{\sigma_{i o} \mathrm{v}_{e}} / \mathrm{v}_{0}$ with respect to $s$, and to proceed by identification. Equation (39) for $\varpi^{F}$ is obtained by differentiation of $\left\langle f^{F}\right\rangle=\varpi^{S}\left\langle f^{S}\right\rangle$.

\section{APPENDIX C: CUMULANTS OF THE OPTICAL DEPTH}

We derive here Eq. (21) for the $r$ th order cumulant of the optical depth $\tau=\int_{0}^{s} \nu d s^{\prime}$, when density fluctuations follow the multivariate Gamma distribution defined in Sec. II B. By definition of the MGD, ${ }^{27}$ we have

$$
\tau=\sum_{i=1}^{M} \int_{0}^{s} g_{i}^{2} d s
$$

where the $g_{i}$ follow multivariate Gaussian statistics, with $\left\langle\left\langle g_{i}\left(s_{1}\right) g_{j}\left(s_{2}\right)\right\rangle\right\rangle=G\left(s_{1}, s_{2}\right) \delta_{i j}$. Here, $G$ is the Gaussian covariance function (from which the covariance matrix $\mathbf{G}$ in Eq. (12) is determined). We next use the Karhunen-Loeve representation $^{55}$ for $g_{i}(s)$, namely

$$
g(s)=\sum_{j=1}^{+\infty} \sqrt{e_{j}} \xi_{j} \phi_{j}(s)
$$

where the $\xi_{j}$ are independent normal variables, and the $e_{j}$ and $\phi_{j}(s)$ are, respectively, the eigenvalues and eigenvectors of the covariance, defined through

$$
\int_{0}^{s} d s_{2} G\left(s_{1}, s_{2}\right) \phi_{i}\left(s_{2}\right)=e_{i} \phi_{i}\left(s_{1}\right),
$$

with $\int d s \phi_{i}(s) \phi_{j}(s)=\delta_{i j}$. Note that the eigenvalues are functions of $s$. Combining Eqs. (C1) and (C2), we obtain the following result for the cumulant generating function $\psi(u)$ of the optical depth

$$
\psi(u)=\ln \left\langle e^{-u \tau(s)}\right\rangle=-\frac{M}{2} \sum_{j=1}^{+\infty} \ln \left(1+2 e_{j}(s) u\right) .
$$

Note that this result could have been obtained also by diagonalizing the covariance matrix $(s / k) \mathbf{G}$ in Eq. (12) (the prefactor $s / k$ ensures that the trace of the covariance matrix is $s)$, and using $\psi(u)=\ln Z\left[\mathbf{u}_{1}\right]$ with $\mathbf{u}_{1}=(u, u, \ldots, u)^{\dagger}$, finally letting $k \rightarrow+\infty$. The cumulant of order $r$ is then given by

$$
\left\langle\left\langle\tau^{r}\right\rangle\right\rangle=\left.(-1)^{r} \frac{d^{r} \psi}{d u^{r}}\right|_{u=0}=2^{r-1}(r-1) ! M \sum_{j=1}^{+\infty} e_{j}^{r} .
$$

The last step is to express $\sum_{j=1}^{+\infty} e_{j}^{r}$ in terms of the correlation function. This is done by iterating Eq. (C3) $r$ times

$$
\begin{aligned}
& \int_{0}^{s} d s_{1} \int_{0}^{s} d s_{2} \ldots \int_{0}^{s} d s_{r} G\left(s_{1}, s_{2}\right) \ldots G\left(s_{r-1}, r_{n}\right) \phi_{j}\left(r_{n}\right) \\
& \quad=e_{j}^{r-1} \phi_{j}\left(s_{1}\right) .
\end{aligned}
$$

The final step makes use of Mercer's theorem, ${ }^{34}$ that is

$$
G\left(s_{r}, s_{1}\right)=\sum_{j=1}^{+\infty} e_{j} \phi_{j}\left(s_{r}\right) \phi_{j}\left(s_{1}\right)
$$

Equation (C6) is multiplied by $e_{j} \phi_{j}\left(s_{1}\right)$ and integrated over $s_{1}$, which yields

$$
\sum_{j=1}^{+\infty} e_{j}^{r}=\int_{0}^{s} d s_{1} \int_{0}^{s} d s_{2} \ldots \int_{0}^{s} d s_{r} G\left(s_{1}, s_{2}\right) \ldots G\left(s_{r-1}, s_{r}\right) G\left(s_{r}, s_{1}\right) .
$$

Equation (21) follows from Eqs. (C8), (C5), and (28).

It should be noted that the cumulant expansion, which is a Taylor series expansion around $u=0$, has a finite convergence radius for the statistics considered here. In fact, expanding the logarithms around $u=0$ in Eq. (C4) yields Eq. (C5) for the cumulants, provided $2 e_{\max }(s) u<1$, where $e_{\max }$ is the largest of the eigenvalues of the operator $G$. The average we are interested in is obtained by setting $u=1$ in Eq. (C4) so that the convergence condition for the cumulant expansion can be stated as $e_{\max }(s)<1 / 2$. For an exponential covariance function, $e_{\max }$ can be calculated from simple 
formulas. Following, e.g., Ref. 55, differentiating Eq. (C3) twice with respect to $s_{1}$ leads to the following differential equation:

$$
\frac{d^{2} f}{d s_{1}^{2}}+\omega_{n}^{2} f\left(s_{1}\right)=0
$$

where $\omega_{n}$ is given by a transcendental equation

$$
\tan \left(\omega_{n} s\right)=\frac{4 \lambda_{L} \omega_{n}}{4\left(\lambda_{L} \omega_{n}\right)^{2}-1},
$$

which takes into account the proper integration domain (from 0 to $s$, as in Ref. 52). The eigenvalues are then given by

$$
e_{n}=\frac{1}{\beta \ell} \frac{2 \lambda_{L}}{1+4\left(\lambda_{L} \omega_{n}\right)^{2}},
$$

and the largest one corresponds to the smallest $\omega_{n}$.

\section{APPENDIX D: DERIVATION OF THE IONIZATION COEFFICIENT IN THE FAST RECYCLING CASE}

We start from the following relation for the derivative of the characteristic function $Z(\mathbf{u})$ of the MGD proved in Ref. 22:

$$
\frac{\partial Z}{\partial u_{k}}=-\beta \frac{1-\left(A^{-1}\right)_{k k}}{u_{k}} Z(\mathbf{u}),
$$

using $\partial_{u_{k}}|A|=|A|\left(1-\left(A^{-1}\right)_{k k}\right) / u_{k}$. In order to differentiate Eq. (1) with respect to $u_{1}$, we note that $\left(A^{-1}\right)_{k k}=\mathscr{C}_{k k} /|A|$, where $\mathscr{C}_{k k}$ is a cofactor. In the notations of Sec. III, $\mathscr{C}_{k k}=|B|$. As a result, it is straightforward to show that

$$
\frac{\partial\left(A^{-1}\right)_{k k}}{\partial u_{1}}=\left(A^{-1}\right)_{k k} \frac{\left(A^{-1}\right)_{11}-\left(B^{-1}\right)_{11}}{u_{1}} .
$$

Then finally,

$$
\frac{\partial^{2} Z}{\partial u_{1} \partial u_{k}}=-\left[\beta \frac{1-\left(A^{-1}\right)_{k k}}{u_{k}}+\left(A^{-1}\right)_{k k} \frac{\left(A^{-1}\right)_{11}-\left(B^{-1}\right)_{11}}{u_{k}\left(1-\left(A^{-1}\right)_{11}\right)}\right] \frac{\partial Z}{\partial u_{1}},
$$

from which Eq. (38) follows. Note that in the discrete limit, all the $u_{j}$ are taken equal to $\epsilon \overline{\sigma_{i o} \mathrm{~V}_{e}} / \mathrm{v}_{0}$, where $\epsilon=\left|\mathbf{r}-\mathbf{r}_{w}\right| / k$ is the discretization step.

\footnotetext{
${ }^{1}$ A. S. Kukushkin, H. D. Pacher, V. Kotov, G. W. Pacher, and D. Reiter, Fusion Eng. Des. 86, 2865 (2011).

${ }^{2}$ D. Reiter, M. Baelmans, and P. Boerner, Fusion Sci. Technol. 47(2), 172 (2005).

${ }^{3}$ T. D. Rognlien, J. L. Milovich, M. E. Rensink, and G. D. Porter, J. Nucl. Mater. 196-198, 347 (1992).

${ }^{4}$ T. D. Rognlien and M. E. Rensink, Fusion Eng. Des. 60, 497 (2002).

${ }^{5}$ H. Bufferand, B. Bensiali, G. Ciraolo, Ph. Ghendrih, P. Genesio, Y. Marandet, A. Paredes, F. Schwander, E. Serre, and P. Tamain, "Near wall plasma simulations using the penalization technique with transport code SOLEDGE2D-EIRENE," J. Nucl. Mater (submitted).
}

${ }^{6}$ A. Loarte, B. Lipschultz, A. S. Kukushkin, G. F. Matthews, P. C. Stangeby, N. Asakura, G. F. Counsell, G. Federici, A. Kallenbach, K. Krieger, A. Mahdavi, V. Phillips, D. Reiter, J. Roth, J. Strachan, D. Whyte, R. Doerner, T. Eich, W. Fundamenski, A. Herrmann, M. Fenstermacher, P. Ghendrih, M. Groth, A. Kirschner, S. Konoshima, B. LaBombard, P. Lang, A. W. Leonard, P. Monier-Garbet, R. Neu, H. Pacher, B. Pégourié, R. A. Pitts, S. Takamura, J. Terry, E. Tsitrone, and ITPA Scrape-off layer and Divertor Physics Topical Group, Nucl. Fusion 47, S203 (2007).

${ }^{7}$ Y. Sarazin and Ph. Ghendrih, Phys. Plasmas 5, 4214-4228 (1998).

${ }^{8}$ A. Yu. Pigarov, S. I. Krasheninnikov, T. D. Rognlien, M. J. Schaffer, and W. P. West, Phys. Plasmas 9, 1287 (2002).

${ }^{9}$ J. A. Boedo, J. Nucl. Mater. 390-391, 29 (2009).

${ }^{10}$ Y. Marandet, H. Capes, L. Godbert-Mouret, M. Koubiti, J. Rosato, and R. Stamm, Eur. Phys. J. D 39, 247 (2006).

${ }^{11}$ Y. Marandet, J. Rosato, H. Capes, L. Godbert-Mouret, M. Koubiti, A. Mekkaoui, F. B. Rosmej, and R. Stamm, High Energy Density Phys. 5, 312 (2009).

${ }^{12}$ S. I. Krasheninnikov, A. Yu. Pigarov, T. K. Soboleva, and D. L. Rudakov, Phys. Plasmas 16, 014501 (2009).

${ }^{13}$ E. Havlickova, W. Fundamenski, V. Naulin, A. H. Nielsen, S. Wiesen, J. Horacek, and J. Seidl, J. Nucl. Mater. 415, S471 (2011).

${ }^{14}$ J. Rosato, H. Capes, F. Catoire, M. B. Kadomtsev, M. G. Levashova, V. S. Lisitsa, Y. Marandet, F. B. Rosmej, and R. Stamm, J. Nucl. Mater. 415, S617 (2011).

${ }^{15}$ J. Rosato, F. Catoire, Y. Marandet, A. Mekkaoui, M. Koubiti, R. Stamm, M. B. Kadomtsev, M. G. Levashova, V. S. Lisitsa, and F. B. Rosmej, Phys. Lett. A 375, 4187 (2011).

${ }^{16}$ F. Catoire, H. Capes, Y. Marandet, A. Mekkaoui, J. Rosato M. Koubiti, and R. Stamm, Phys. Rev. A 83, 012518 (2011).

${ }^{17}$ A. Yu. Pigarov, S. I. Krasheninnikov, and T. D. Rognlien, Phys. Plasmas 18, 092503 (2011).

${ }^{18}$ A. Yu. Pigarov, S. I. Krasheninnikov, and T. D. Rognlien, Phys. Plasmas 19, 072516 (2012).

${ }^{19}$ A. Mekkaoui, Y. Marandet, D. Reiter, P. Boerner, F. Catoire, P. Genesio, J. Rosato, H. Capes, F. Catoire, L. Godbert-Mouret, M. Koubiti, and R. Stamm, Phys. Plasmas 19, 060701 (2012).

${ }^{20}$ A. Mekkaoui, Y. Marandet, H. Capes, D. Reiter, L. Godbert-Mouret, M. Koubiti, J. Rosato, and R. Stamm, Transp. Theor. Stat. Phys. 41, 71 (2012).

${ }^{21}$ G. C. Pomraning, Linear Kinetic Theory and Particle Transport in Stochastic Mixtures (World Scientific, 1991).

${ }^{22}$ Y. Marandet, A. Mekkaoui, D. Reiter, P. Boerner, P. Genesio, J. Rosato, H. Capes, F. Catoire, M. Koubiti, L. Godbert-Mouret, and R. Stamm, Plasma Phys. Controlled Fusion 53, 065001 (2011).

${ }^{23}$ Y. Marandet, A. Mekkaoui, D. Reiter, P. Boerner, P. Genesio, F. Catoire, J. Rosato, R. Stamm, H. Capes, M. Koubiti, and L. Godbert-Mouret, Nucl. Fusion 51, 083035 (2011).

${ }^{24}$ J. P. Graves, J. Horacek, R. A. Pitts, and K. I. Hopcraft, Plasma Phys. Controlled Fusion 47, L1 (2005).

${ }^{25}$ J. A. Krommes, Phys. Plasmas 15, 030703 (2008).

${ }^{26}$ O. E. Garcia, Phys. Rev. Lett. 108, 265001 (2012).

${ }^{27}$ P. R. Krishnaiah and M. M. Rao, Am. Math. Monthly 68, 342 (1961).

${ }^{28}$ A. Huber, U. Samm, B. Schweer, and Ph. Mertens, Plasma Phys. Controlled Fusion 47, 409-440 (2005).

${ }^{29}$ E. Lukacs, Characteristic Functions (Charles Griffin, London, 1960).

${ }^{30}$ S. Kotz, N. Balakrishnan, and N. L. Johnson, Continuous Multivariate Distributions (Wiley, 2000).

${ }^{31}$ R. C. Griffiths, J. Multivariate Anal. 15, 13 (1984).

${ }^{32}$ J. A. Krommes, Phys. Rep. 360, 1 (2002).

${ }^{33}$ A. M. Yaglom, Correlation Theory of Stationary and Related Random Functions. Vol I: Basic Results (Springer-Verlag, 1981).

${ }^{34}$ U. Grenander and G. Szegö, Toeplitz Forms and Their Applications (University of California Press, Berkeley, 1958).

${ }^{35}$ A. Kolovos, G. Christakos, D. T. Hristopulos, and M. L. Serre, Adv. Water Resour. 27, 815 (2004).

${ }^{36}$ N. Cressie and H. C. Huang, J. Am. Stat. Assoc. 94, 1330 (1999).

${ }^{37}$ D. T. Hristopulos, Stochastic Environmental Research and Risk Assessment 16, 43 (2002)

${ }^{38}$ G. Christakos, Water Resour. Res. 20, 251, doi:10.1029/WR020i002p00251 (1984).

${ }^{39}$ C. Berg, J. P. R. Christensen, and P. Ressel, Harmonic Analysis on Semigroups, Theory of Positive Definite and Related Functions (SpringerVerlag, 1984).

${ }^{40}$ C. Ma, Stat. Probab. Lett. 61, 411 (2003).

${ }^{41}$ N. G. Van Kampen, Stochastic Processes in Physics and Chemistry, 2nd ed. (Elsevier, 1992). 
${ }^{42}$ B. Silberman and A. Boettcher, Introduction to Large Truncated Toeplitz Matrices (Springer-Verlag, New York, 1999).

${ }^{43}$ R. Kubo, J. Math. Phys. 4, 174-183 (1963).

${ }^{44}$ A. Brissaud and U. Frisch, J. Math. Phys. 15, 524 (1974).

${ }^{45}$ N. Fedorczak, J. P. Gunn, J. Y. Pascal, Ph. Ghendrih, Y. Marandet, and P. Monier-Garbet, Phys. Plasmas 19, 072313 (2012).

${ }^{46} \mathrm{P}$. C. Stangeby, The Plasma Boundary of Magnetic Fusion Devices (Taylor \& Francis, New York, 2000).

${ }^{47}$ R. K. Janev, W. D. Langer, K. Evans, and D. E. Post, Elementary Processes in Hydrogen-Helium Plasmas (Springer-Verlag, 1987).
${ }^{48}$ H. P. Summers, The ADAS User Manual (2004), http://www.adas.ac.uk/. ${ }^{49}$ F. Sattin, N. Vianello, and M. Valisa, Phys. Plasmas 11, 5032 (2004).

${ }^{50}$ R. Kubo, J. Phys. Soc. Jpn. 17, 1100 (1962).

${ }^{51}$ R. Meeron, J. Chem. Phys. 27, 1238 (1957).

${ }^{52}$ E. D. Fichtl and A. K. Prinja, J. Quant. Spectrosc. Radiat. Transf. 112, 646 (2011).

${ }^{53}$ R. B. Leipnik, J. Aust. Math. Soc. Ser. B, Appl. Math. 32, 327-347 (1991).

${ }^{54}$ T. Yoneda, J. Math. Anal. Appl. 332, 487-496 (2007).

${ }^{55}$ R. G. Ghanem and P. D. Spanos, Stochastic Finite Elements, A Spectral Approach (Dover, Mineola, NY, 1991). 